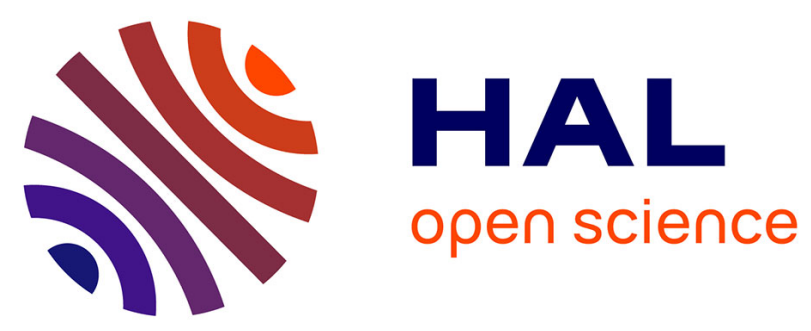

\title{
Accelerating cardiac diffusion tensor imaging combining local low-rank and 3D TV constraint
}

\author{
J. P. Huang, L.H. Wang, C.Y. Chu, Liu W. Y., Y. M. Zhu
}

\section{To cite this version:}

J. P. Huang, L.H. Wang, C.Y. Chu, Liu W. Y., Y. M. Zhu. Accelerating cardiac diffusion tensor imaging combining local low-rank and 3D TV constraint. Magnetic Resonance Materials in Physics, Biology and Medicine, 2019, 32 (4), pp.407-422. 10.1007/s10334-019-00747-1 . hal-02073170

\section{HAL Id: hal-02073170 https://hal.science/hal-02073170}

Submitted on 17 Dec 2020

HAL is a multi-disciplinary open access archive for the deposit and dissemination of scientific research documents, whether they are published or not. The documents may come from teaching and research institutions in France or abroad, or from public or private research centers.
L'archive ouverte pluridisciplinaire HAL, est destinée au dépôt et à la diffusion de documents scientifiques de niveau recherche, publiés ou non, émanant des établissements d'enseignement et de recherche français ou étrangers, des laboratoires publics ou privés. 


\title{
Accelerating Cardiac Diffusion Tensor Imaging Combining Local Low-Rank and 3D TV Constraint
}

\author{
Jianping Huang ${ }^{1,2,5}$, Lihui Wang ${ }^{3}$, Chunyu $\mathrm{Chu}^{4}$, Wanyu $\mathrm{Liu}^{2}$, and Yuemin $\mathrm{Zhu}^{2,5}$ \\ ${ }^{1}$ College of Mechanical and Electrical Engineering, Northeast Forestry University, Heilongjiang, Harbin 150040, China \\ ${ }^{2}$ Metislab, LIA CNRS, Harbin Institute of Technology, Harbin, Heilongjiang, 150001, China \\ ${ }^{3}$ Key Laboratory of Intelligent Medical Image Analysis and Precise Diagnosis of Guizhou Province, \\ School of Computer Science and Technology, Guizhou University, Guiyang, China \\ ${ }^{4}$ College of Engineering, Bohai University, Jinzhou 121013, China \\ ${ }^{5}$ CREATIS, CNRS UMR5220, Inserm U1206, INSA Lyon, University of Lyon, Lyon, France \\ E-mail: Jianping Huang, jianping829@gmail.com;
}

\begin{abstract}
Object: Diffusion tensor magnetic resonance imaging (DT-MRI, or DTI) is a promising technique for invasively probing biological tissue structures. However, DTI is known to suffer from much longer acquisition time with respect to conventional MRI and the problem is worsened when dealing with in vivo acquisitions. Therefore, faster DTI for both ex vivo and in vivo scans is highly desired.

Materials and Methods: This paper proposes a new compressed sensing (CS) reconstruction method that employs local low-rank (LLR) model and three-dimensional (3D) total variation (TV) constraint to reconstruct cardiac diffusion-weighted (DW) images from highly undersampled k-space data. The LLR model takes the set of DW images corresponding to different diffusion gradient directions as a 3D image volume and decomposes the latter into overlapping 3D blocks. Then, the 3D blocks are stacked as two-dimensional (2D) matrix. Finally, low-rank property is applied to each block matrix and the $3 \mathrm{D}$ TV constraint to the 3D image volume. The underlying constrained optimization problem is finally solved using the first-order fast method. The proposed method is evaluated on real ex vivo cardiac DTI data as a prerequisite to in vivo cardiac DTI applications.

Results: The results on real human ex vivo cardiac DTI images demonstrate that the proposed method exhibits lower reconstruction errors for DTI indices, including fractional anisotropy (FA), mean diffusivities (MD), transverse angle (TA) and helix angle (HA), compared to existing CS-based DTI image reconstruction techniques.

Conclusion: The proposed method provides better reconstruction quality and more accurate DTI indices in comparison with the state-of-the-art CS-based DW image reconstruction methods.

Keywords: Cardiac Diffusion Tensor Imaging, Compressed sensing, Sparse sampling, Constrained reconstruction, Locally low-rank regularization.
\end{abstract}




\section{Introduction}

Diffusion tensor magnetic resonance imaging (DT-MRI, or DTI) is an MRI technique that allows for investigating noninvasively the fiber architectures of the human heart [1-11]. However, cardiac DTI is known to suffer from long acquisition time to obtain high quality images (in terms of temporal resolution, spatial resolution, and signal-to-noise-ratio-SNR) for both ex vivo [12-16] and in vivo [17-19] hearts. This is because DTI requires, with respect to conventional MRI, supplementary acquisitions at different (at least six) diffusion gradient directions. The problem is worsened when dealing with in vivo DTI acquisitions since patient movement and cardiac motion often cause image quality degradation and motion artifacts [20-24]. Therefore, shortening acquisition time is highly desired for both ex vivo and in vivo DTI. It becomes indispensable in reducing motion artifacts and improving image quality for clinical cardiac DTI. Many attempts have been made to shorten the acquisition time by reducing the amount of acquisition data, such as parallel imaging [25-29], partial k-space imaging [30,31], and simultaneous multi-slice (SMS) imaging [32,33].

In recent years, compressed sensing (CS) has emerged as a new framework for reconstructing signals with high quality from less measurements than the traditional Shannon-Nyquist sampling theorem [34-36]. CS exploits sparsity or compressibility of signals in certain domain (pixel or transform domain) and combines sampling and compression into a unified framework. To date, CS has been successfully applied to biomedical imaging [37,38], and has shown great potential for MRI [39-41] and computed tomography (CT) [42-44].

In light of the time-reducing potential of CS and considering that the diffusion-weighted (DW) images obtained along different diffusion gradient directions of DTI are often correlated, a constrained image reconstruction technique for cardiac DTI based on a regularization framework was proposed [45], in which the total variation (TV) constraint was chosen in both spatial domain and diffusion direction to accelerate DTI acquisitions. Both sparsity prior and intra- and inter-correlation of DW images were also embedded in a single constraint term in the CS extensions (distributed CS) to further reduce DTI acquisition time [46]. Utilizing the inter-image correlation of DW images, the combination of CS and parallel imaging further improved acquisition efficiency [47]. By stacking the DW images as column vectors of a matrix, the resulting matrix is then rank-deficient, which allowed the authors of $[48,49]$ to formulate the CS-based DTI image reconstruction from undersampled k-space as a low-rank matrix approximation problem. Observing that phase changes drastically across diffusion gradient directions, a phase-constrained low-rank (PCLR) approach was developed 
[50]. In [51-53], model-based CS methods for DTI were proposed, which use the signal intensity model to directly estimate diffusion tensor fields from undersampled k-space data.

Meanwhile, local low-rank (LLR) model has been recently proposed in various medical imaging applications such as quantitative water-fat MRI [54], dynamic MRI image reconstruction [55] and MRI parameter mapping [56]. Compared to global low-rank (GLR) methods that capture global correlation among whole images, the LLR model is more suitable for accounting for local information. The present study proposes a new CS reconstruction method that employs both LLR model and three-dimensional (3D) TV constraint to reconstruct cardiac DTI images. The LLR model takes the set of DW images corresponding to different diffusion gradient directions as a 3D volume and decomposes the latter into overlapping 3D blocks. Then, the 3D blocks are stacked as two-dimensional (2D) matrix. Finally, low-rank property is applied to each block matrix and the 3D TV constraint to the 3D image volume. The underlying constrained optimization problem is finally solved using the first-order fast method. The proposed method is evaluated on real ex vivo cardiac DTI data as a prerequisite to in vivo cardiac DTI applications.

The rest of the paper is organized as follows. In Section 2, the mathematical formulation of the proposed method and experimental settings are given. Then, in Section 3, the proposed method is evaluated on human ex vivo heart DW images and compared to the state-of-the-art methods. Finally, the discussion and conclusion are given in Sections 4 and 5, respectively.

\section{Materials and methods}

\subsection{Proposed method}

Assuming that $\mathrm{x}_{l}$ is a vectorised DW image and $F_{l}^{u}$ is a partial Fourier transform for $l^{\text {th }}$ diffusion direction. The undersampled k-space data $\mathrm{y}_{l}$ of the DW image for each direction in $\mathrm{k}$-space can then be formulated as:

$$
\mathrm{y}_{l}=F_{l}^{u} \mathrm{x}_{l}+\varepsilon_{l},
$$

where $\varepsilon_{l}$ is the observed noise and $l=1,2, \ldots, L$ the diffusion direction, and $F_{l}^{u}=P \cdot F$ with $F$ denoting Fourier transform and $P$ the undersampling pattern (mask). Note that in practical acquisitions, raw k-space data are always complex and non-Hermitian symmetric. As a result, the corresponding spatial DW image $\mathrm{x}_{l}$ is always complex and contains a spatial phase. 
All the undersampled k-space data $Y$ of the DW images in all the diffusion gradient directions can be written as:

$$
Y=F^{u} \cdot X+\varepsilon,
$$

where $Y=\left[\mathrm{y}_{1}, \mathrm{y}_{2}, \ldots, \mathrm{y}_{L}\right], \quad X=\left[\mathrm{x}_{1}, \mathrm{x}_{2}, \ldots, \mathrm{x}_{L}\right]$ whose columns represent the vectorised DW image, $\varepsilon=\left[\varepsilon_{1}, \varepsilon_{2}, \ldots, \varepsilon_{L}\right]$, and $F^{u}$ is a partial Fourier transform for all the diffusion directions and is defined as:

$$
F^{u}=\left[\begin{array}{ccc}
F_{1}^{u} & & 0 \\
& \ddots & \\
0 & & F_{L}^{u}
\end{array}\right] .
$$

The DW images are sparse/compressible in certain domain such as wavelet domain. In addition, since the DW images acquired in different diffusion gradient directions have similar anatomical structures, they are somewhat correlated. Consequently, by stacking these images as column vectors of a matrix $X$, the latter will be low rank. Then, the reconstruction of DW images from undersampled k-space data is performed by solving the following optimization problem (i.e. GLR model, as illustrated in figure 1(a)):

$$
X=\arg \min _{X}\left\{\frac{1}{2}\left\|F^{u} X-Y\right\|_{2}^{2}+\tau\|X\|_{*}+\lambda \cdot\|T \cdot X\|_{1}\right\}
$$

where $\left\|F^{u} X-Y\right\|_{2}^{2}$ represents data fidelity term, $\|X\|_{*}$ is the nuclear norm or sum of singular values of the matrix $X,\|T \cdot X\|_{1}$ is the $l 1$-norm of $X$ with sparsity transform $T$, and $\tau>0$ and $\lambda>0$ are the regularization parameters.

However, as mentioned before, GLR models involving entire images capture only globally correlated information in the image. We are then led to use the LLR model to account for local image information. As illustrated in figure 1(b), the LLR model-based method includes the following main steps: a) consider the set of DW images corresponding to different diffusion gradient directions as a 3D image volume; b) divide the 3D image volume into overlapping 3D image blocks of size $n_{x} \times n_{y} \times n_{d}$ with $n_{d}$ indicating the size in the diffusion gradient direction; c) stack each 2D DW image block of size $n_{x} \times n_{y}$ as a column vector of a 2D matrix, thus forming a block matrix of size $n_{x} n_{y} \times n_{d} \quad\left(b_{i}\right.$ matrix in figure 1(b)); d) apply low-rank property (singular values shrinkage operator) to the block matrix; e) reconstruct the DW images $X$ by concatenating all the block matrices. 

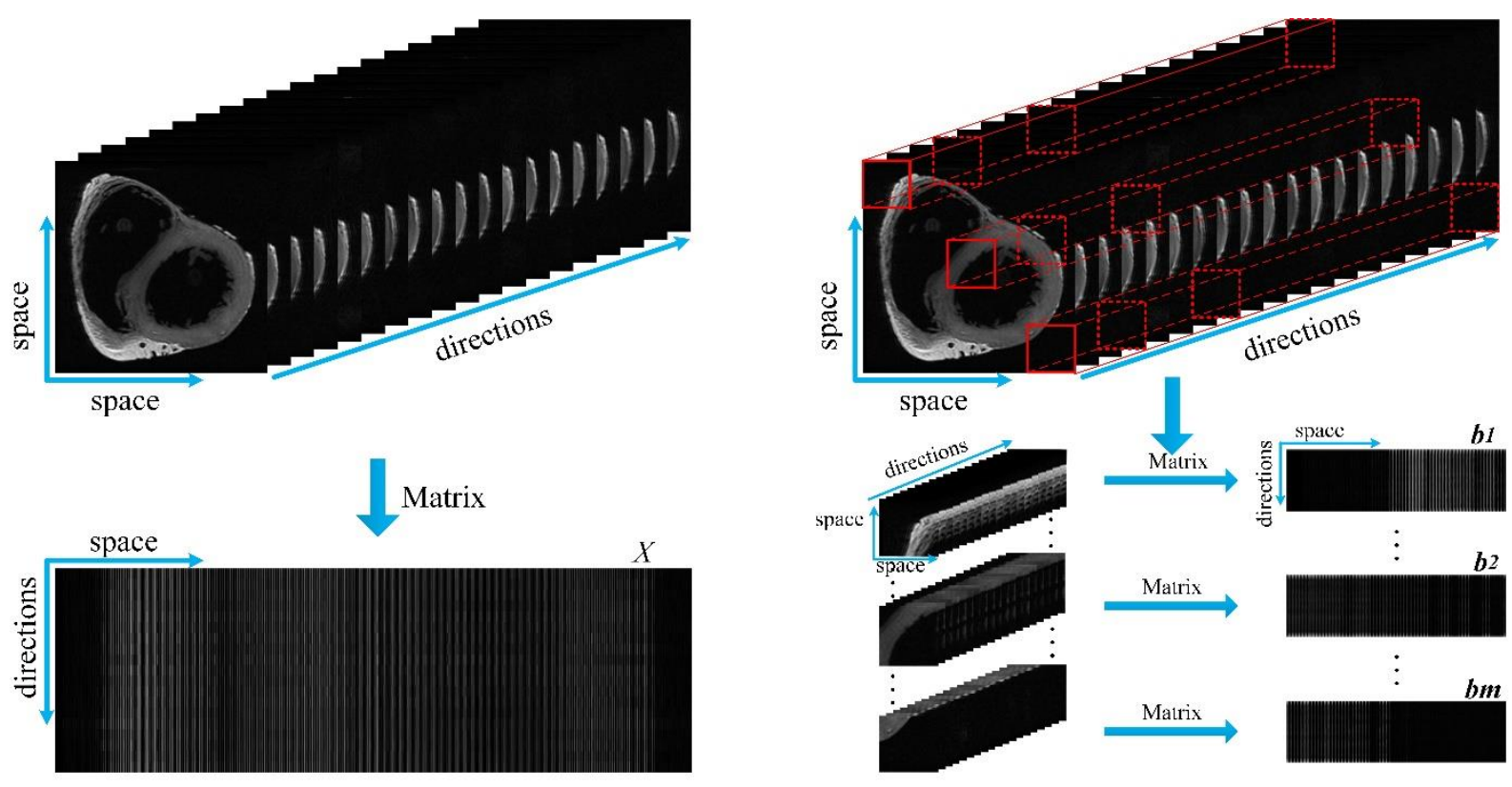

a

b

Fig. 1 Illustration of the principle of global low-rank (GLR) and local low-rank (LLR) methods. In the GLR matrix $X$, each column corresponds to an entire DW image and the number of its columns is equal to the number of diffusion gradient directions $L$.

Using the above-described LLR method, the reconstruction then becomes the following optimization problem:

$$
X=\arg \min _{X}\left\{\frac{1}{2}\left\|F^{u} X-Y\right\|_{2}^{2}+\tau \sum_{b \in \Omega}\left\|R_{b} X\right\|_{*}+\lambda \cdot \operatorname{TV} 3 D(X)\right\},
$$

where $F^{u}$ is the partial Fourier transform for all diffusion directions as defined in $(3), R_{b}$ is the operator that extracts the $b^{\text {th }}$ block, $\Omega$ designates the set of blocks, $\operatorname{TV} 3 D(\cdot)$ is the TV regularization term, which can either be the anisotropic or the isotropic TV norm. In this paper, we choose the isotropic TV defined as $\operatorname{TV} 3 D(X)=\sum_{i, j, k}\left\|D_{i, j, k} X\right\|_{2}=\sum_{i, j, k} \sqrt{\left(X_{i, j, k}-X_{i+1, j, k}\right)^{2}+\left(X_{i, j, k}-X_{i, j+1, k}\right)^{2}+\left(X_{i, j, k}-X_{i, j, k+1}\right)^{2}}$, where $D_{i, j, k}$ means the forward finite-difference operators along the horizontal, vertical, and diffusion gradient directions of DW images, respectively.

This problem can be effectively solved using the Fast Composite Splitting Algorithm (FCSA) introduced in [57]. This method is based on the combination of both variable and operator splitting techniques, which decompose the regularization problem (5) into two simpler regularization subproblems. The main steps of this method is as follows: (a) splitting variable $x$ into two variables 
$\left\{x_{i}\right\}_{i=1,2}$; (b) performing operator splitting to minimize total variation regularization and nuclear norm regularization subproblems over $\left\{x_{i}\right\}_{i=1,2}$ respectively, and (c) obtaining the solution $x$ by linear combination of $\left\{x_{i}\right\}_{i=1,2}$. Let $f(X)=\frac{1}{2}\left\|F^{u} X-Y\right\|_{2}^{2}$ that is a convex and smooth function with the Lipschitz constant $L_{f}, \quad g_{1}(X)=\tau \sum_{b \in \Omega}\left\|R_{b} X\right\|_{*}$ that is a convex but non-smooth function and $g_{2}(X)=\lambda \cdot \operatorname{TV} 3 D(X)$. Then, the $g(X)=g_{1}(X)+g_{2}(X)$ problem can be divided into two subproblems: nuclear norm regularization and TV-norm regularization. Each subproblem is actually a convex function that can be solved by a proximal mapping operation [58]: $X_{1}^{k}=\operatorname{prox}_{\rho}\left(\tau \sum_{b \in \Omega}\left\|R_{b} X\right\|_{*}\right)\left(x_{g}\right)$ and $X_{2}^{k}=\operatorname{prox}_{\rho}\left(2 \lambda\|X\|_{T V 3 D}\right)\left(x_{g}\right)$. According to [58], given a continuous convex function $g(x)$ and any scalar $q>0$, the proximal map associated with function $g$ is defined as: $\operatorname{prox}_{\rho}(\phi)(x)=\underset{u}{\arg \min }\left\{\phi(u)+\frac{1}{2 \rho}\|u-x\|_{2}^{2}\right\}$, where $\rho$ is the inverse of the Lipschitz constant $L_{f}$ having $\nabla f=\left(\frac{1}{2}\left\|F^{u} X-Y\right\|_{2}^{2}\right)^{\prime}=\left(F^{u}\right)^{T}\left(F^{u} X-Y\right)$ with $\left(F^{u}\right)^{T}$ indicating the inverse partial Fourier transform.

The reconstruction problem (5) is outlined in the following algorithm.

\section{INPUT:}

$K:$ the maximum number of iterations;

$\tau, \lambda:$ the regularization parameters;

$n_{x}, n_{y}, n_{d}$ : the block sizes;

tol: the tolerance parameter.

INIT: $\rho=1 / L, t^{1}=1, X^{0}=r^{1}=0, k=0$;

\section{REPEAT:}

$k=k+1$;

$x_{g}=r^{k}-\rho \nabla f\left(r^{k}\right)$;

$X_{1}^{k}=\operatorname{prox}_{\rho}\left(\tau \sum_{b \in \Omega}\left\|R_{b} X\right\|_{*}\right)\left(x_{g}\right) ;$

$X_{2}^{k}=\operatorname{prox}_{\rho}\left(2 \lambda\|X\|_{T V 3 D}\right)\left(x_{g}\right)$;

$X^{k}=\frac{X_{1}^{k}+X_{2}^{k}}{2}$; 


$$
\begin{aligned}
& X^{k}=a b s\left(X^{k}\right) ; \\
& t^{k+1}=\frac{1+\sqrt{1+4\left(t^{k}\right)^{2}}}{2} ; \\
& r^{k+1}=X^{k}+\frac{t^{k-1}-1}{t^{k}}\left(X^{k}-X^{k-1}\right) ; \\
& \text { UNTIL } k>K \text { OR } \frac{\left\|X^{k-1}-X^{k}\right\|_{2}}{\left\|X^{k}\right\|_{2}}<\text { tol } .
\end{aligned}
$$

\section{OUTPUT:}

$$
X=a b s\left(X^{k}\right): \text { the reconstructed DW images. }
$$

\subsection{Experimental data}

The real DW data correspond to ex vivo samples of the human hearts $[59,12,60]$ from the website http://cvrgrid.org/data/ex-vivo. The corresponding acquisition parameters are the following: image size $=256 \times 256 \times 134$, image spatial resolution $=0.43 \times 0.43 \times 1.0 \mathrm{~mm}^{3}$, and number of diffusion gradient directions 21. In the present study, seven slices of the image volume have been used to evaluate the proposed method (the $b_{0}$ image of the $5^{\text {th }}$ slice was shown in figure $2(a)$ ). We used the same k-space sampling patterns as those used in [61,62], which concern Cartesian undersampling patterns with one-dimensional (1D) variable density phase-encode random undersampling (i.e. in $k_{y}$ direction) and $2 \mathrm{D}$ variable density random undersampling pattern. An example of the $1 \mathrm{D}$ and $2 \mathrm{D}$ random k-space undersampling patterns is shown in figure 2(b) and figure 2(c), in which the sampling ratio (R) was set to $15 \%$.

The above initial DW data are in fact spatial magnitude data (so, their corresponding k-space data are complex but Hermitian symmetric) while in practice DW data are always spatial complex because the acquired raw k-space data are always complex and non-Hermitian symmetric. To mimic actual acquisitions, we have rendered the magnitude DW data complex in the following manner: a) take the Fourier transform of spatial magnitude DW data, providing complex but Hermitian-symmetric k-space data; b) undersample the complex k-space data, thus making the k-space data asymmetric; c) add complex Gaussian white noise to the asymmetric k-space data, rendering them even more asymmetric; d) take the inverse Fourier transform of the undersampled noisy k-space data, leading to complex spatial DW data containing phase information. 


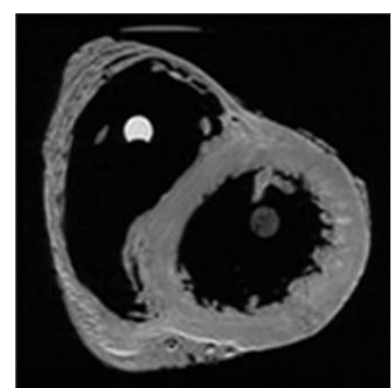

$\mathbf{a}$

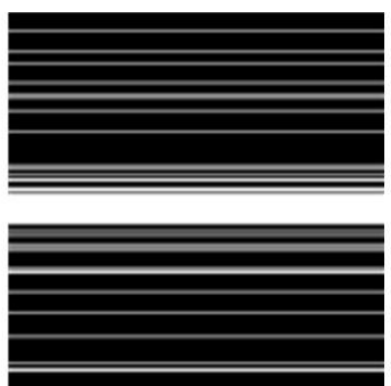

b

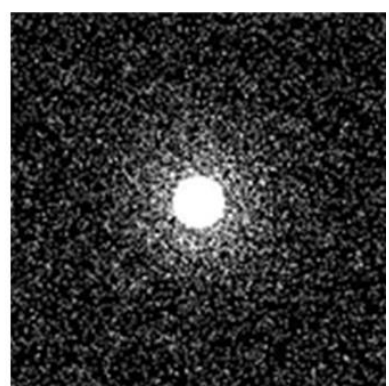

c

Fig. 2 Example of (a) the $b_{0}$ image of the $5^{\text {th }}$ slice, (b) $1 \mathrm{D}$ random k-space undersampling mask and (c) 2D variable density random k-space undersampling pattern with a sampling ratio of $15 \%$ (i.e. keeping $15 \%$ of the full k-space data).

\subsection{Evaluation}

To evaluate the performance of the proposed method (LLR+TV3D), comparison was performed with zero-filling (replace the non-acquired k-space data by zeros and then inverse Fourier transform the zero-filled k-space data), joint sparsity (JS) [46], JS with TV3D (JS+TV3D), global low-rank (GLR) [48], GLR with TV3D (GLR+TV3D) and, local low-rank without TV3D (LLR) methods.

In addition to qualitative assessment consisting of visually comparing reconstruction results, several quantitative indices were calculated for the DW images reconstructed with different methods. They are the fractional anisotropy (FA), mean diffusivity (MD), helix angle (HA) and transverse angle (TA) $[63,7]$.

The MD characterizes the mean diffusivity of the tissue:

$$
\mathrm{MD}=\frac{\lambda_{1}+\lambda_{2}+\lambda_{3}}{3}
$$

The FA is used to describe the diffusion anisotropy of the tissue:

$$
\mathrm{FA}=\sqrt{\frac{3 \cdot\left[\left(\lambda_{1}-\mathrm{MD}\right)^{2}+\left(\lambda_{2}-\mathrm{MD}\right)^{2}+\left(\lambda_{3}-\mathrm{MD}\right)^{2}\right]}{2 \cdot\left(\lambda_{1}^{2}+\lambda_{2}^{2}+\lambda_{3}^{2}\right)}} .
$$

The HA is defined as the angle between the projection of the primary eigenvector onto the tangent plane and the imaging plane, and the TA is defined as the angle between the projection of the primary eigenvector onto the imaging plane and the tangent plane [63,7]. The pair (HA, TA) describes completely fiber orientation.

To quantitatively compare different reconstruction methods, the root mean square errors (RMSE) was first calculated for each slice according to:

$$
\mathrm{RMSE}=\sqrt{\frac{\left\|\operatorname{vec}\left(x_{\text {rec }}\right)-\operatorname{vec}\left(x_{\text {ref }}\right)\right\|_{2}^{2}}{N}},
$$


where $x_{r e c}$ and $x_{\text {ref }}$ denote respectively the calculated quantity and the reference quantity, and $N$ the total number of signals. Then, the mean RMSE (mRMSE) values of FA, MD, TA and HA were calculated to evaluate and compare the reconstruction performance.

The observation measurement $Y$ is corrupted by complex Gaussian white noise $\varepsilon$ with standard deviation $\sigma_{n}$. The associated input SNR (ISNR) [64] is defined as ISNR $=20 \log _{10}\left(\frac{\sigma_{x}}{\sigma_{n}}\right)$ with $\sigma_{x}$ denoting the standard deviation of the reference image.

\subsection{Parameter setting}

In the following experiments, 1D variable density phase-encode random undersampling was used. The ISNR was set to $20 \mathrm{~dB}$. The Daubechies wavelets with four decomposition levels were used. The threshold parameter $\tau$ in soft-thresholding operator for each 3D block was set in an adaptive manner using Stein's unbiased risk estimate (SURE) thresholding [65]. The regularization parameter $\lambda$ was set to 0.1 and the $3 \mathrm{D}$ block size $n_{x} \times n_{y} \times n_{d}$ was set $8 \times 8 \times 21$, respectively.

\section{Results}

\subsection{Visual comparison}

Figures 3 to 6 show the T-DWI, FA, MD, TA and HA maps of $5^{\text {th }}$ slice with the sampling ratio of $25 \%$. In the calculations, the diffusion tensors corresponding to the complete k-space were taken as the references. As observed, the FA, MD, TA and HA maps generated by the proposed method are visually better than those obtained with the other methods.

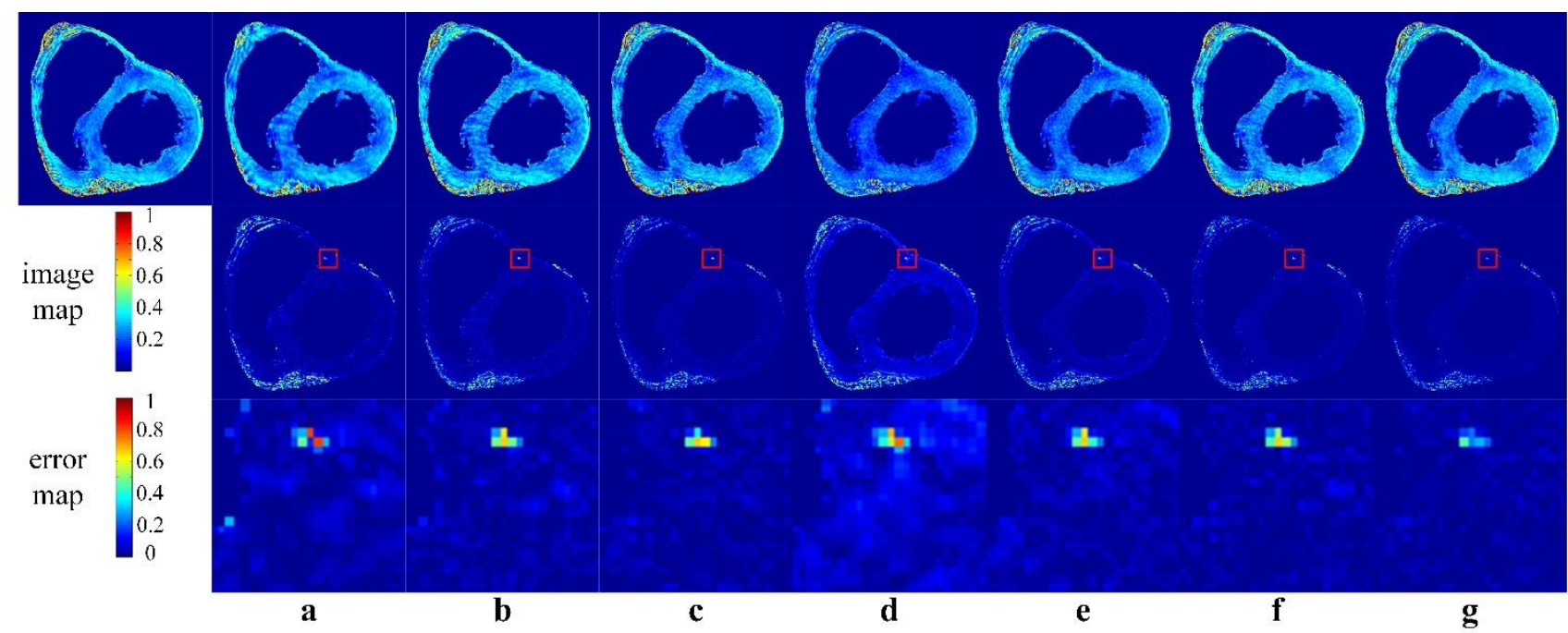

Fig. 3 FA maps of $5^{\text {th }}$ slice. Top row: FA reconstruction maps. Middle row: FA error maps. Bottom row: the ROIs marked by the red boxes in the error maps. The reference FA is shown in the first column of the top row. Reconstructions 
from undersampled k-space (sampling ratio 25\%) using (a) zero-filling method (RMSE=0.0663), (b) JS method (RMSE=0.0529), (c) JS+TV3D method (RMSE=0.0460), (d) GLR method (RMSE=0.0706), (e) GLR+TV3D method $(\mathrm{RMSE}=0.0555)$, (f) LLR method $(\mathrm{RMSE}=0.0459)$ and $(\mathrm{g})$ the proposed method (RMSE=0.0402).

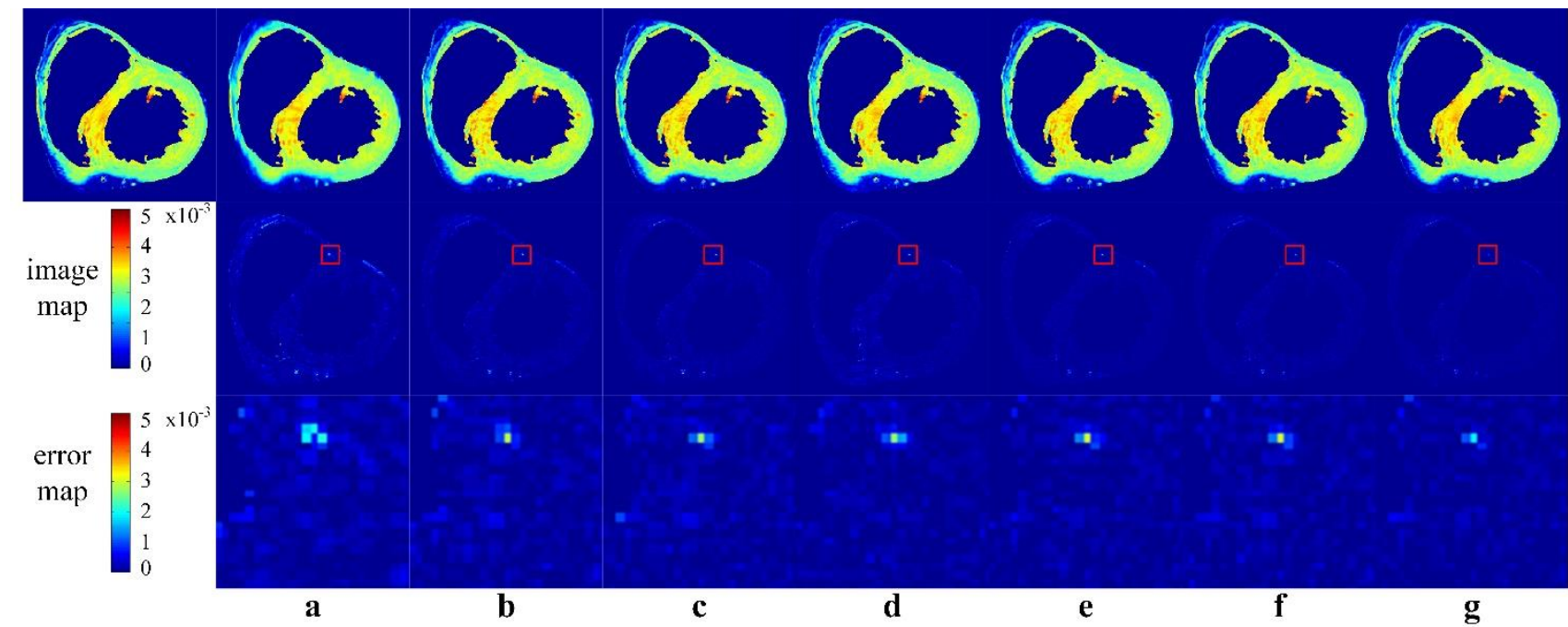

Fig. 4 MD maps of $5^{\text {th }}$ slice. Top row: MD reconstruction maps. Middle row: MD error maps. Bottom row: the ROIs marked by the red boxes in the error maps. The reference MD is shown in the first column of the top row. Reconstructions from undersampled k-space (sampling ratio 25\%) using (a) zero-filling method (RMSE=0.1310), (b) JS method (RMSE=0.0994), (c) JS+TV3D method (RMSE=0.0896), (d) GLR method(RMSE=0.0975), (d) GLR+TV3D method (RMSE=0.0877), (e) LLR method $(\mathrm{RMSE}=0.0868)$ and (f) the proposed method $(\mathrm{RMSE}=0.0748)$.

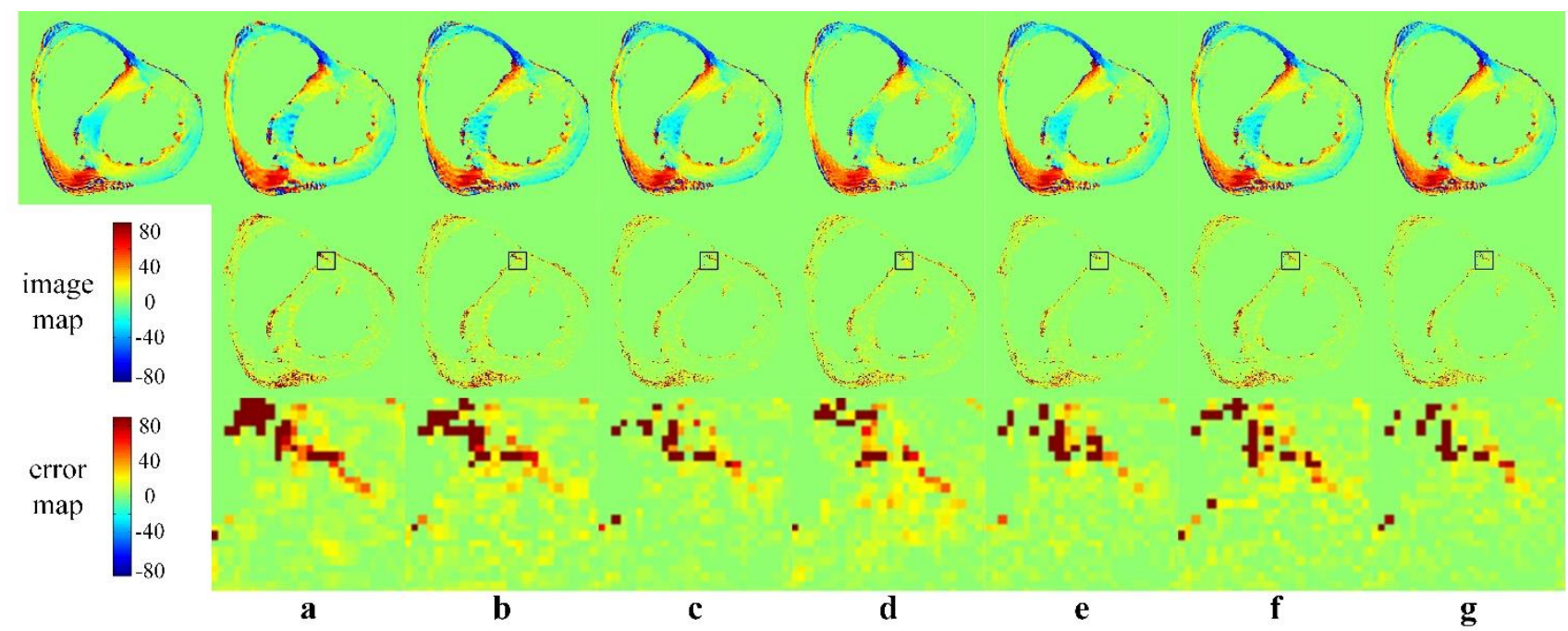

Fig. 5 TA maps of $5^{\text {th }}$ slice. Top row: TA reconstruction maps. Middle row: TA error maps. Bottom row: the ROIs marked by the red boxes the in error maps. The reference TA is shown in the first column of the top row. Reconstructions from undersampled $\mathrm{k}$-space (sampling ratio 25\%) using (a) zero-filling method (RMSE=15.2867), (b) JS method (RMSE =14.7117), (c) JS+TV3D method (RMSE=13.4796), (d) GLR method (RMSE=13.3053), (e) GLR+TV3D method (RMSE=13.1900), (f) LLR method (RMSE=13.7366) and (g) the proposed method (RMSE=12.6375). 


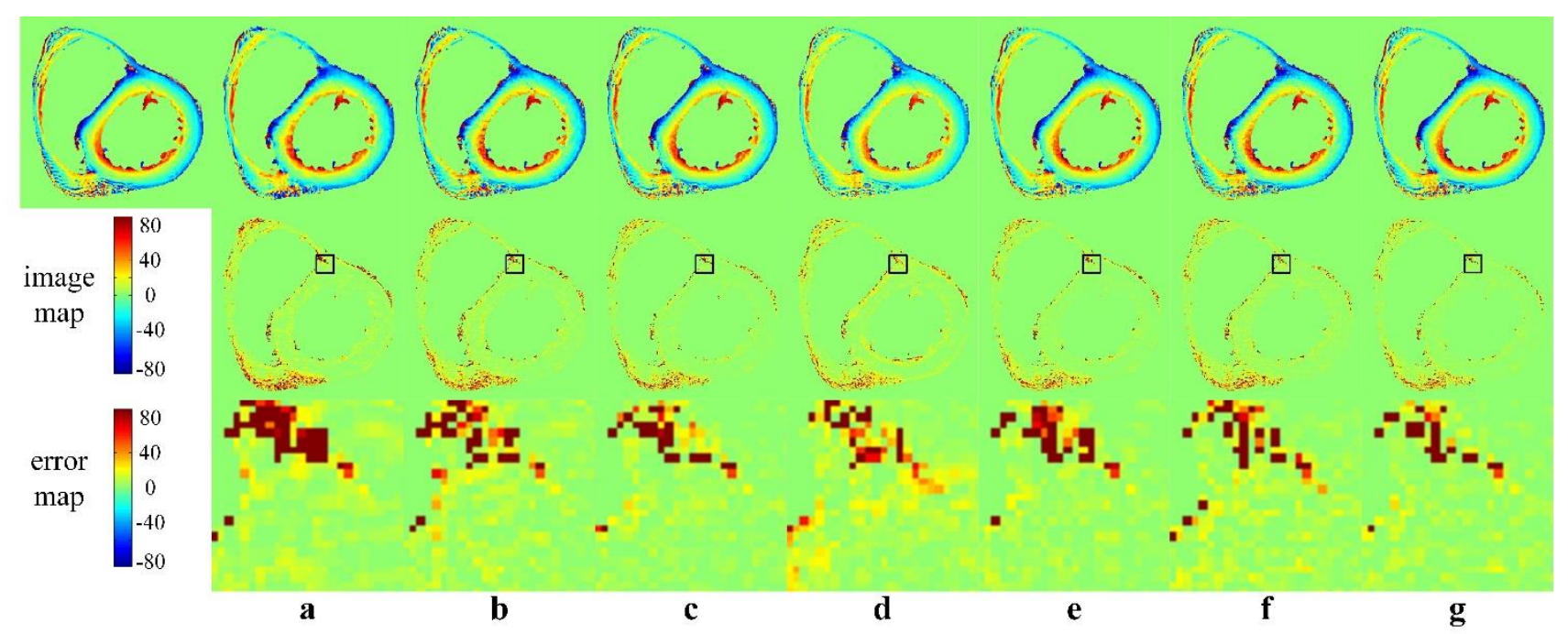

Fig. 6 HA maps of $5^{\text {th }}$ slice. Top row: HA reconstruction maps. Middle row: HA error maps. Bottom row: the ROIs marked by the red boxes in the error maps. The reference HA is shown in the first column of the top row. Reconstructions from undersampled k-space (sampling ratio 25\%) using (a) zero-filling method (RMSE=15.0506), (b) JS method (RMSE =14.1850), (c) JS+TV3D method (RMSE=12.9166), (d) GLR method (RMSE=12.9376), (e) GLR+TV3D method (RMSE=12.5925), (f) LLR method (RMSE=13.0880) and (g) the proposed method (RMSE=11.9483).

\subsection{Sampling ratios}

Figure 7 compares the reconstruction performance (mRMSE of FA, MD, TA and HA) of different methods as a function of sampling ratios ranging from $10 \%$ to $50 \%$. As observed, the proposed method resulted in clearly smaller errors than the other methods for all the quantitative indices.

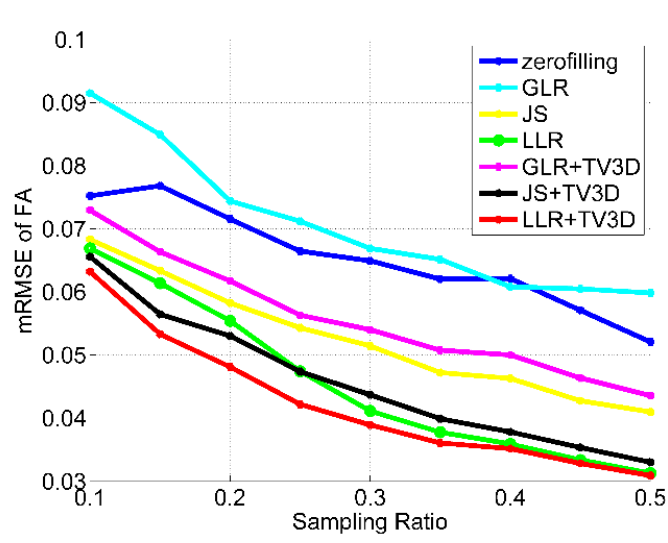

a

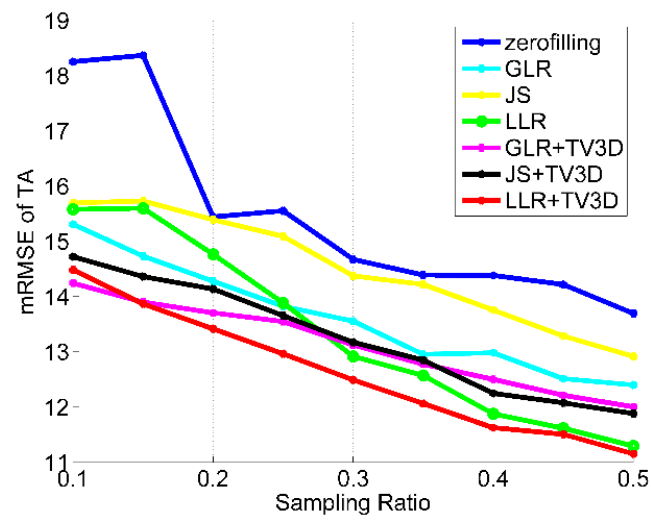

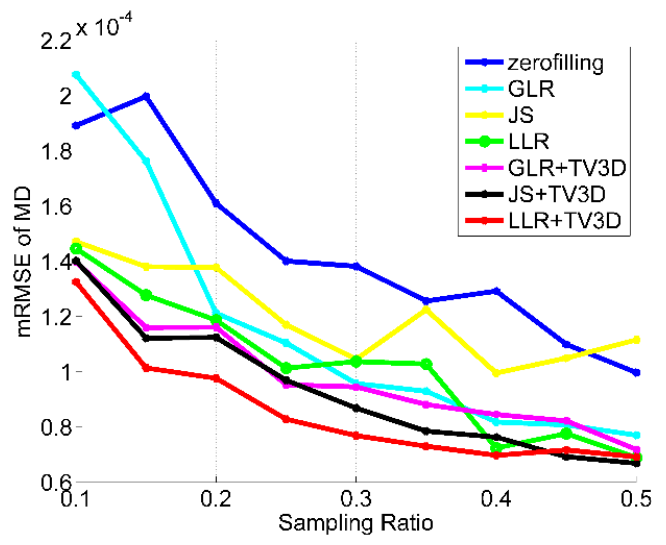

b

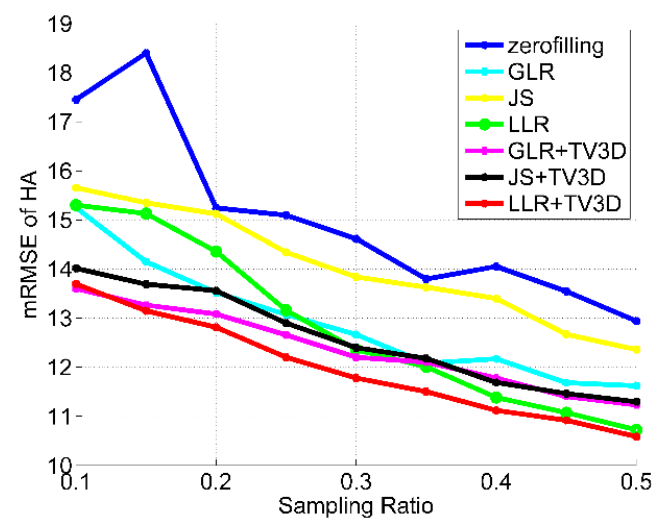


Fig. 7 Performance comparison of different reconstruction methods on real human heart data with different sampling ratios. (a) mRMSE of FA. (b) mRMSE of MD. (c) mRMSE of TA. (d) mRMSE of HA.

\subsection{Robustness to noise}

The performance of reconstruction from undersampled DTI data using the proposed method for different values of ISNR and sampling ratio $(\mathrm{R})$ is illustrated in figure 8 . It is seen that the reconstruction performance changes with the sampling ratio and ISNR. Increasing the sampling ratio reduces the reconstruction error mRMSEs (except in the case ISNR=10dB) and reducing ISNR increases the reconstruction error mRMSEs. For example, when fixing ISNR at $20 \mathrm{~dB}$, a better reconstruction performance is obtained with higher sampling ratios.

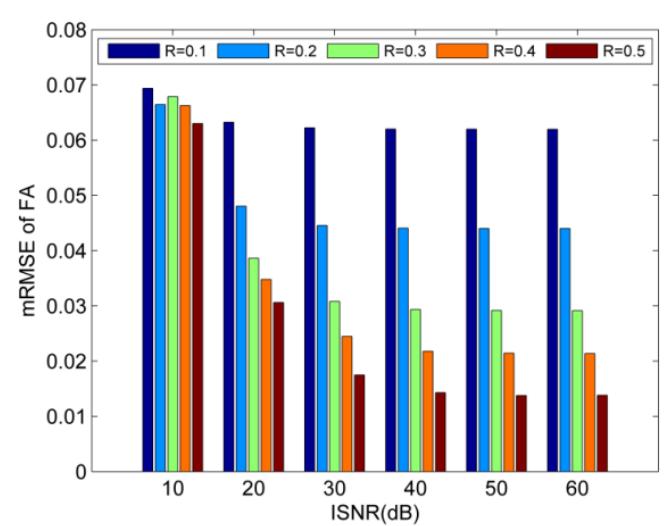

a

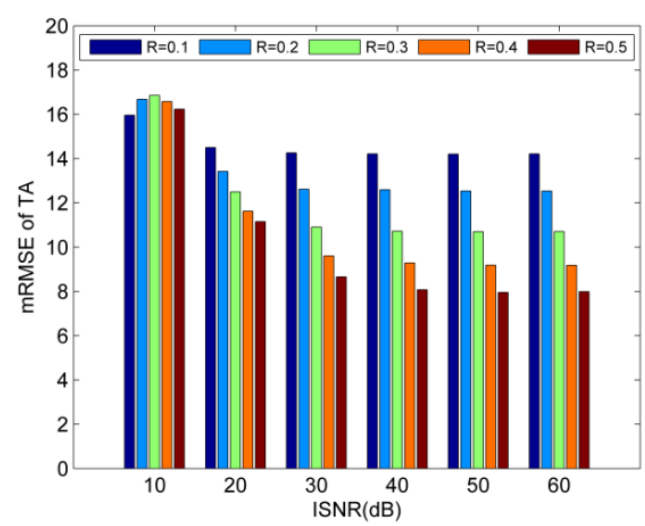

c

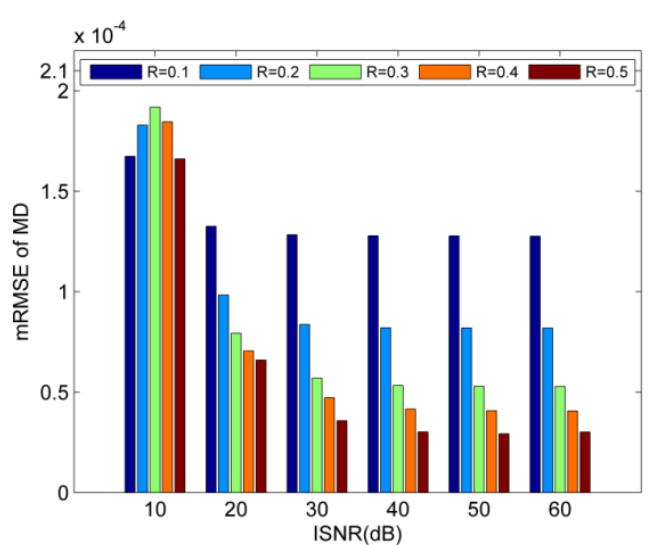

b

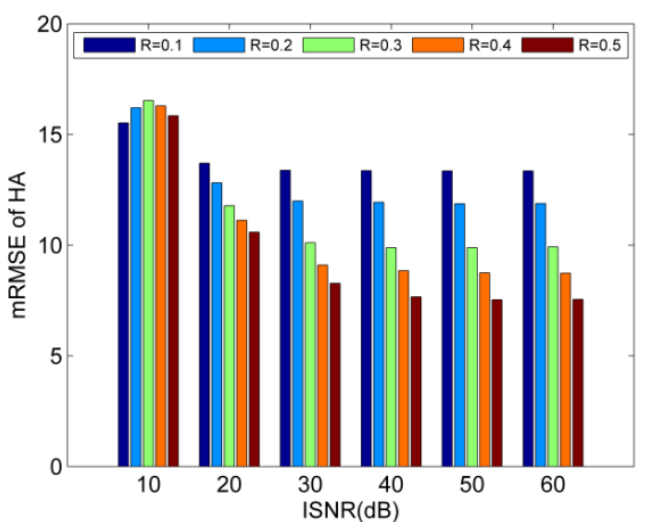

d

Fig. 8 Reconstruction performance of the proposed method with various ISNRs and sampling ratios (Rs) on human heart data. (a) mRMSE of FA. (b) mRMSE of MD. (c) mRMSE of TA. (d) mRMSE of HA.

\subsection{Sampling patterns}

Figure 9 shows the reconstruction performance of the proposed method on two different sampling patterns, namely the 1D variable density random undersampling in phase-encoding direction and 2D variable density random undersampling pattern [61,66], in terms of FA, MD, TA and HA (with sampling ratios from $10 \%$ to $50 \%$ and without noise). As observed, for the same sampling ratio, the 
2D variable density random undersampling resulted in significant reduction of reconstruction errors compared to the $1 \mathrm{D}$ variable density random undersampling.

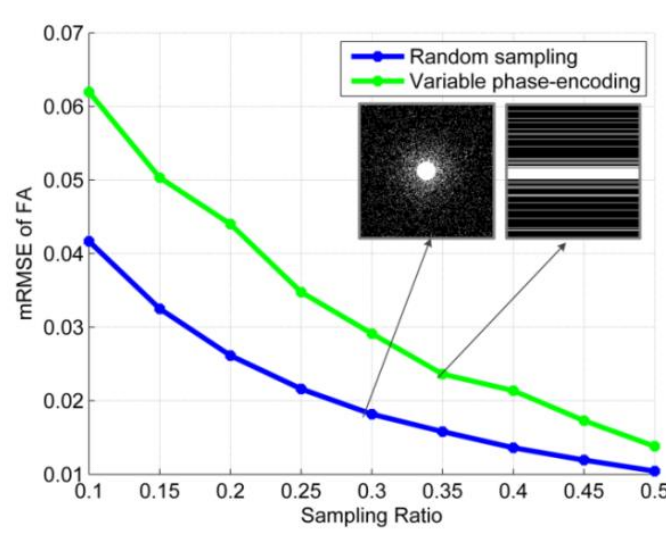

a

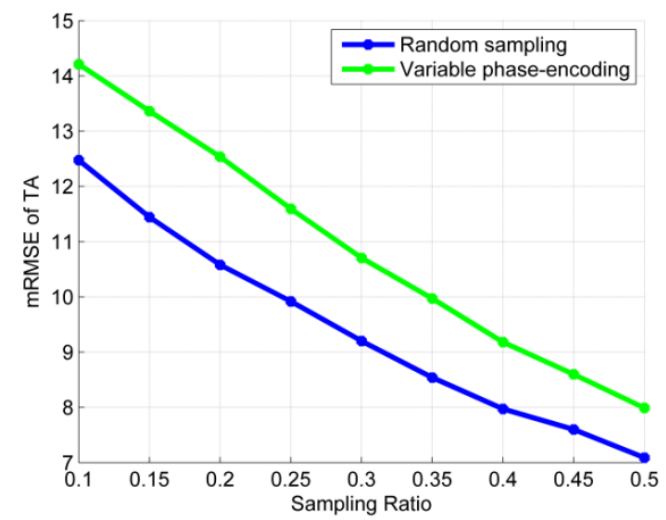

c

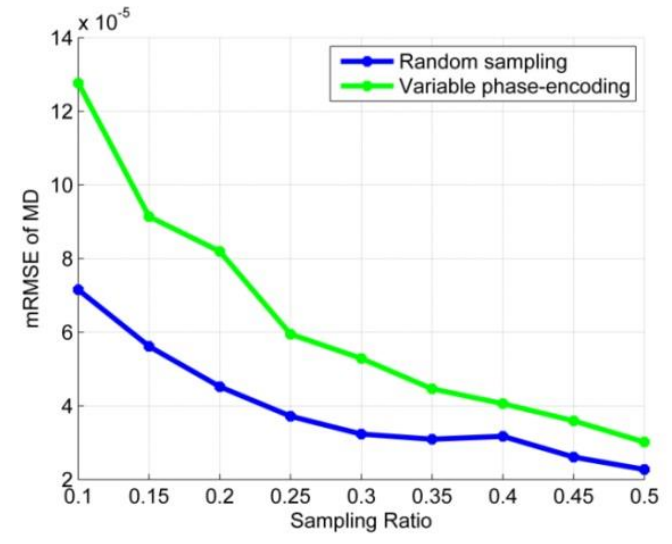

b

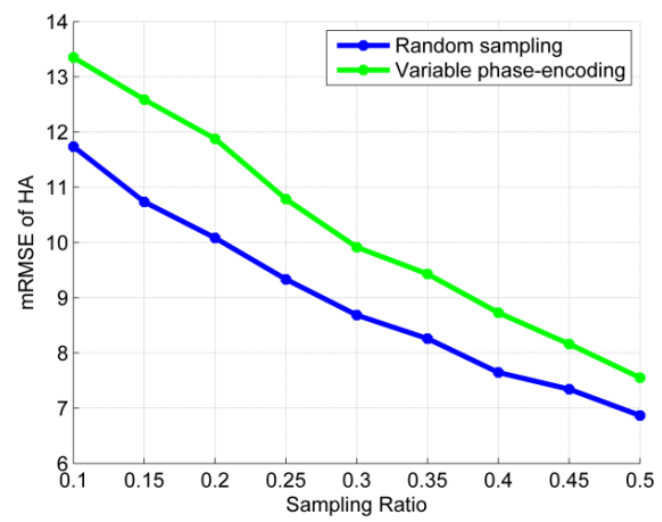

d

Fig. 9 Effect of sampling patterns on (a) mRMSE of FA, (b) mRMSE of MD, (c) mRMSE of TA, and (d) mRMSE of HA.

\subsection{Choice of patch size}

The choice of patch size $\left[n_{x}, n_{y}, n_{d}\right]$ has effects on reconstruction quality. To show that, we calculate the mRMSE values of FA, MD, TA and HA for different $n_{x}$ and $n_{y}$ (fix the number of diffusion directions $n_{d}$ ) and different sampling ratios, as shown in figure 10. Clearly, there exists an optimal value $[n x, n y]=[8,8]$, which gives the smallest mRMSE.

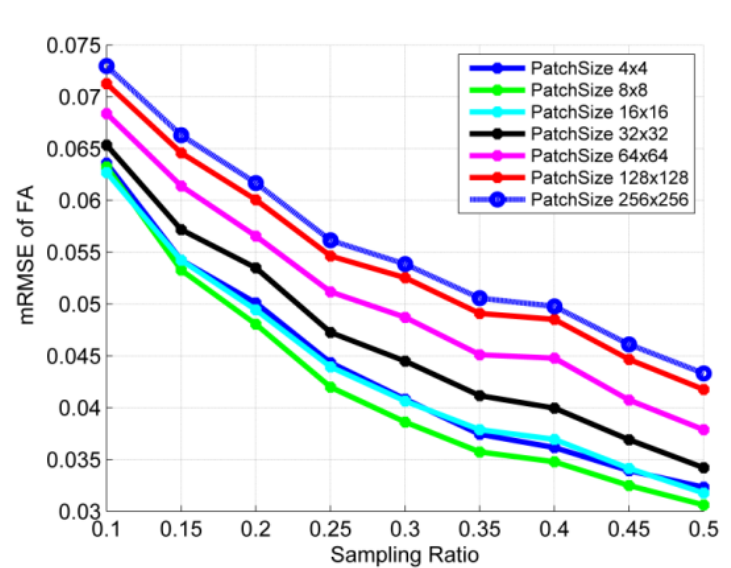

a

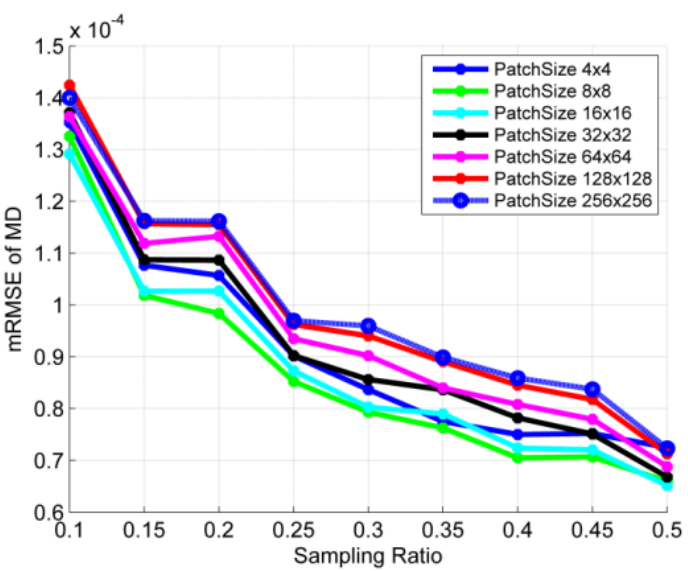

b 


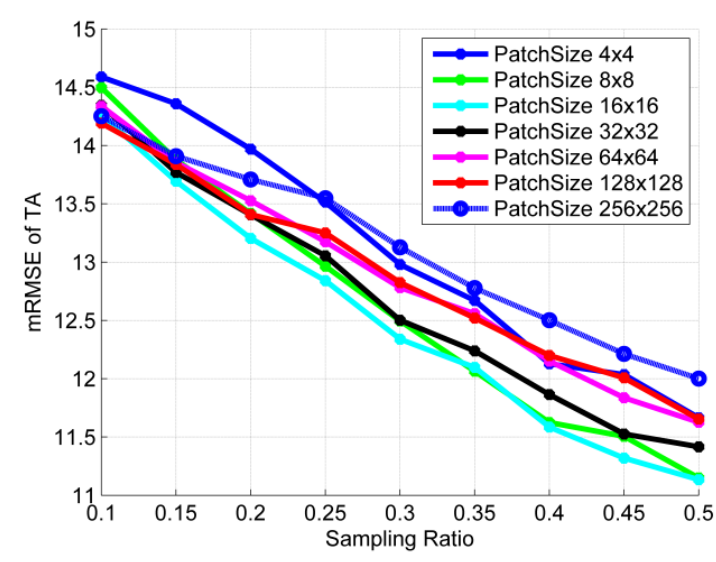

c

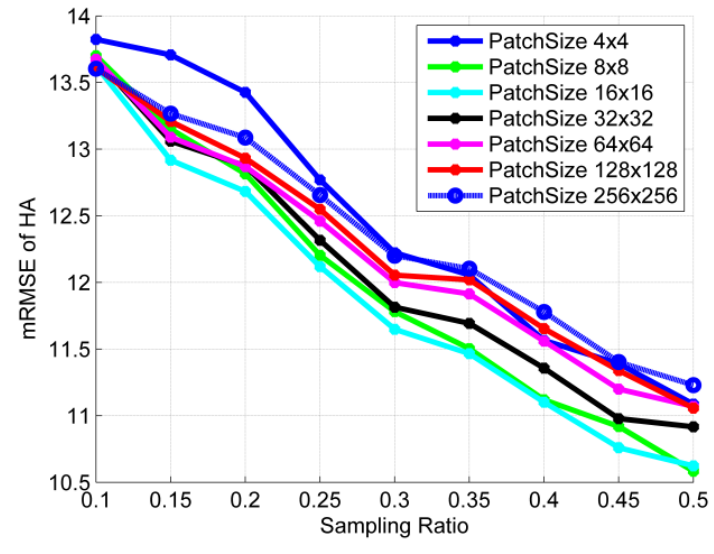

d

Fig. 10 Effect of block sizes on reconstruction quality. (a) mRMSE of FA. (b) mRMSE of MD. (c) mRMSE of TA. (d) mRMSE of HA.

\section{Discussion}

\subsection{Combination of local low-rank and 3D TV constraints}

The comparison of the reconstructions with and without 3D TV constraints shows that the proposed method (with 3D TV) always generated the smallest mRMSE regardless of sampling ratios (figure 7), which means that the reconstruction using the combination of local low-rank penalty and 3D TV penalties clearly reduces reconstruction artifacts and preserves image edges and fine structures. This can be explained as follows. Since the proposed method operates on image blocks, the matrix is more rank-deficient from local image blocks than from 3D image volume. As a result, the use of the LLR allows better reconstructing local image structure information, but may produce excessive edge blurring in the case of high undersampling ratios (e.g. undersamplng ratio of 0.1). Meanwhile, the 3D TV constraint is particularly suitable for reconstructing edges, but tends to over-smooth image details at high undersampling ratios. Hence, the combination of the two constraints has enabled us to achieve better reconstruction quality.

\subsection{Determination of regularization parameter}

The regularization parameter $\lambda$ was empirically determined. It also impacts on reconstruction quality. Figure 11 illustrates the variation of RMSE with different $\lambda$ values on $5^{\text {th }}$ slice with ISNR 20dB. The reconstruction RMSE of FA changes slightly at $\lambda=0.05$ and $\lambda=0.1$ with better performance than the other values (figure 11(a)). On the other hand, the reconstruction RMSE of MD changes slightly when $\lambda$ is larger than 0.05 (figure 11(b)). Therefore, the chosen $\lambda=0.1$ in the present study seemed the best choice. 


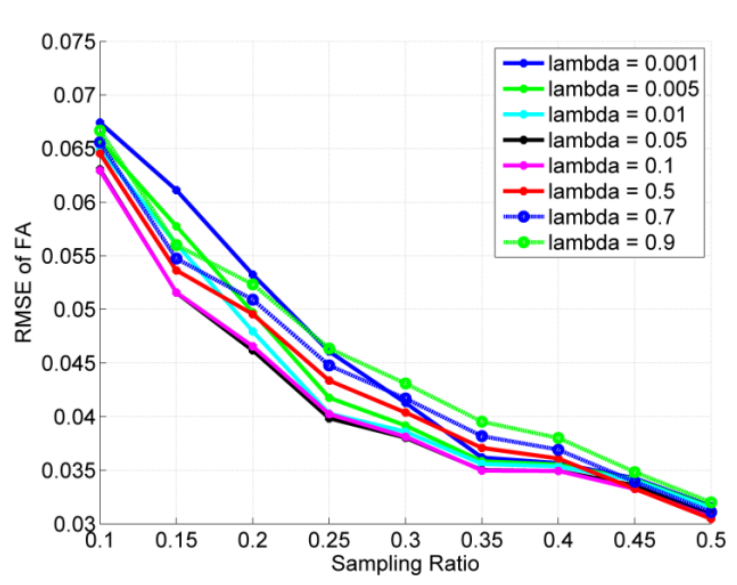

a

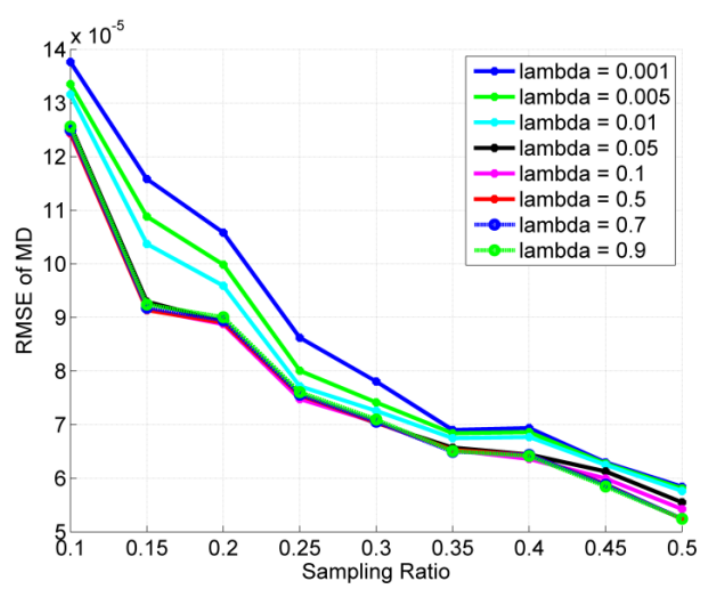

b

Fig. 11 Effect of regularization parameters (DW image of $5^{\text {th }}$ slice, ISNR $20 \mathrm{~dB}$ ). (a) RMSE of FA. (b) RMSE of MD.

\subsection{Effect of sampling patterns}

As observed (figure 9), the 2D variable density random undersampling pattern gives better performance than the $1 \mathrm{D}$ variable density random undersampling pattern. This can be explained as follows. It is well known that when k-space is undersampled, the zero-filled inverse Fourier reconstruction exhibits aliasing artifacts since the Nyquist criterion is violated. The appearance of the aliasing artifacts depends on the undersampling pattern. Equispaced sampling results in coherent folding effects; as a result, image structure overlapping is very visible. In contrast, random or pseudo-random undersampling leads to incoherent interferences $[39,66]$; the final effect of the interferences appears much like additive random noise and consequently, the reconstructed image preserves more initial information. Figure 12 shows an example where the sampling patterns and the corresponding images reconstructed using zero-filling method are given.

Note however that, although ideal, the 2D variable density random undersampling pattern cannot be implemented on current MRI systems whereas the implementation of 1D variable density random undersampling (in phase-encoding direction) scheme is simpler and requires only minor modifications to existing pulse sequences $[61,66]$. 


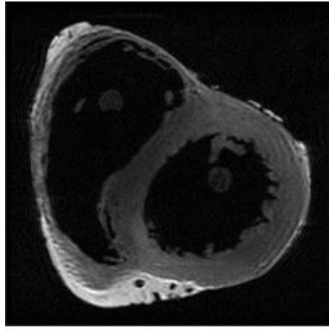

a

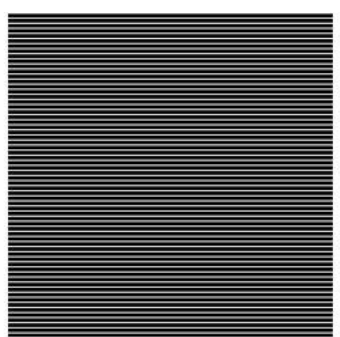

b

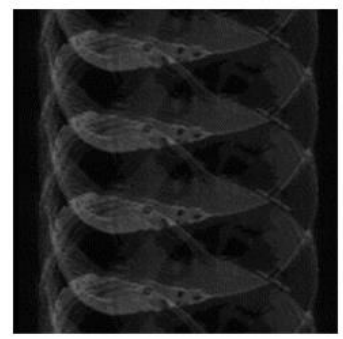

e

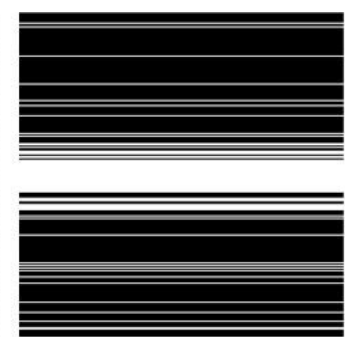

c

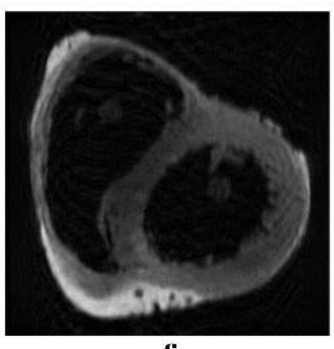

f

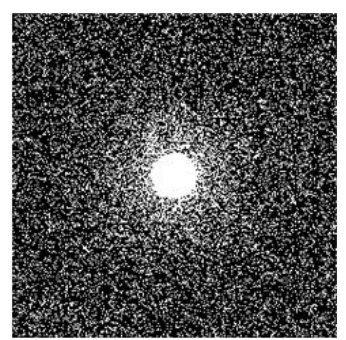

d

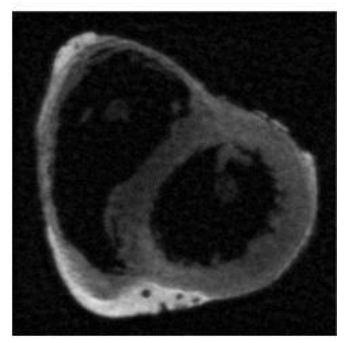

g

Fig. 12 k-space undersampling patterns and the corresponding images reconstructed using zero-filling method. (a) DW image of $5^{\text {th }}$ slice in a given diffusion gradient direction; (b) Equispaced k-space undersampling; (c) 1D variable density random k-space undersampling in phase-encoding direction; (d) 2D variable density random undersampling.

\subsection{Influence of phase information}

In the present study, image reconstruction is formulated as an optimization problem (Eq. 5), in which there are three terms. In each of the three terms, complex images (i.e. phase information) were used. However, we have not used the complex images all the time during the reconstruction process. Indeed, after calculating the above three terms, we used the magnitude of complex image when computing $t^{k+1}=\frac{1+\sqrt{1+4\left(t^{k}\right)^{2}}}{2}$ and $r^{k+1}=X^{k}+\frac{t^{k-1}-1}{t^{k}}\left(X^{k}-X^{k-1}\right)$, in which $X^{k}=a b s\left(X^{k}\right)$. That aimed to simplify the calculation while focusing on the energy of images by taking the magnitude of complex image. It is however possible to use only the magnitude of complex image (by taking $x_{g}=a b s\left(x_{g}\right)$ ) or simply take as such the complex image all the time during the reconstruction, which will then lead to respectively the so-called "Abs" method (no phase information is used) and "Complex" method (phase information is used). Of course, no matter what method we used, the last step is always to take the magnitude of complex image as the final desired image.

The results for ISNR $=0 \mathrm{~dB}$ (noise-free) and ISNR=20 dB are respectively illustrated in Figure 13 and Figure 14.

These results show that, for highly undersampled k-space data (sampling ratio smaller than 15\%), the proposed method gives rise to smaller errors with respect to the cases of using the magnitude of 
complex image or using purely complex image. After certain sampling ratio (saying greater than $20 \%$ ), using the magnitude of complex image leads to the best results while using complex image during the whole reconstruction process yields the worst results. The difference in performance between the three methods can be explained as follows. The "Abs" method always works on the energy (magnitude) of complex image. Since in MRI, images are always produced in such a way that the imaginary part of the complex image is small with respect to the real part, the magnitude image always preserves such importance proportionality during all the calculations. In contrast, with the "Complex" method, the imaginary part has been left to freely and "arbitrarily" vary, which may amplify its weight and thus generate bigger errors. Our proposed method is situated between them with the imaginary part more or less confined.

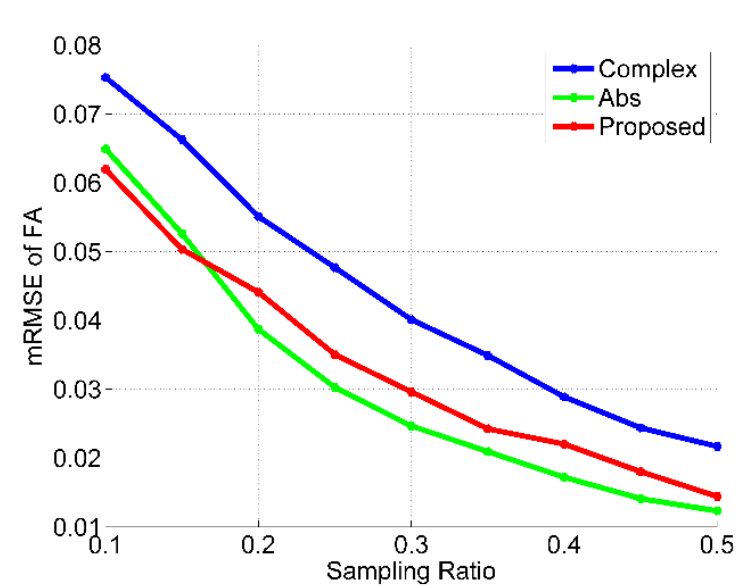

$\mathbf{a}$

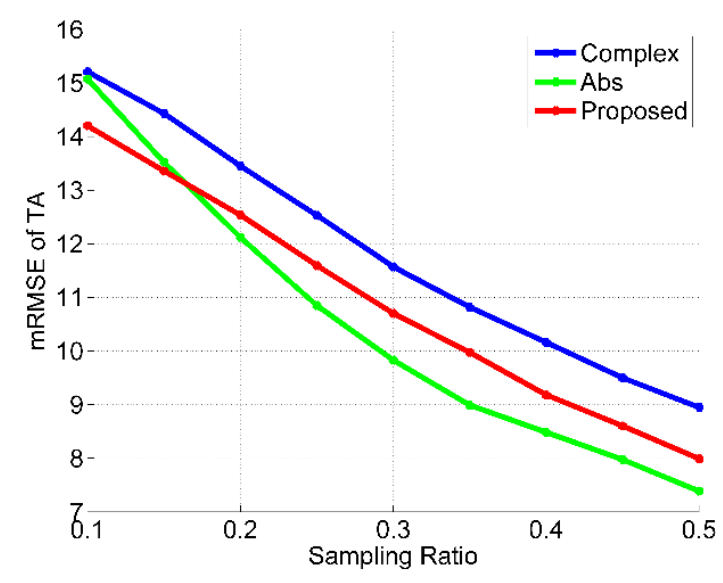

c

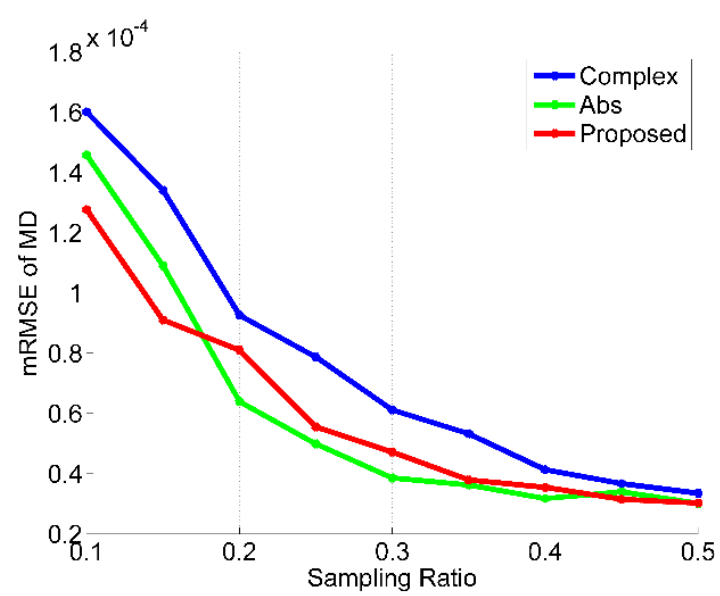

b

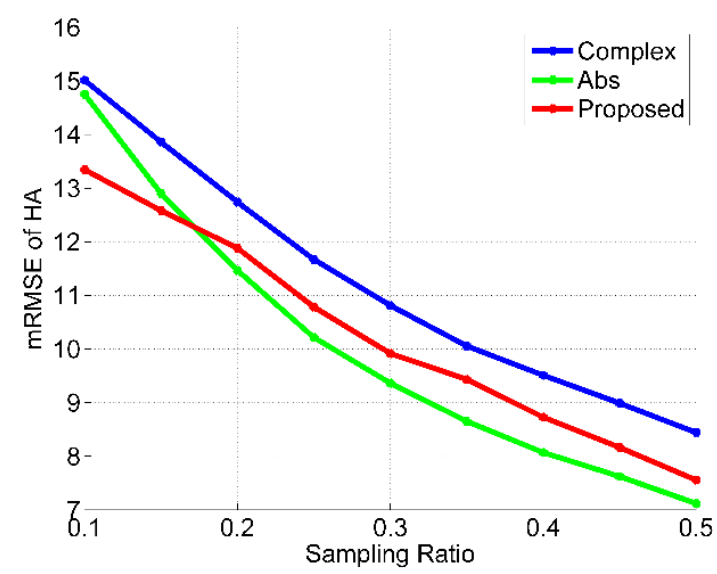

d

Fig. 133 Comparison of reconstructions using or without using phase information for ISNR=0 dB (noise-free). (a) mRMSE of FA, (b) mRMSE of MD, (c) mRMSE of TA, and (d) mRMSE of HA. "Abs" no phase information is used. "Complex": phase information is used. 


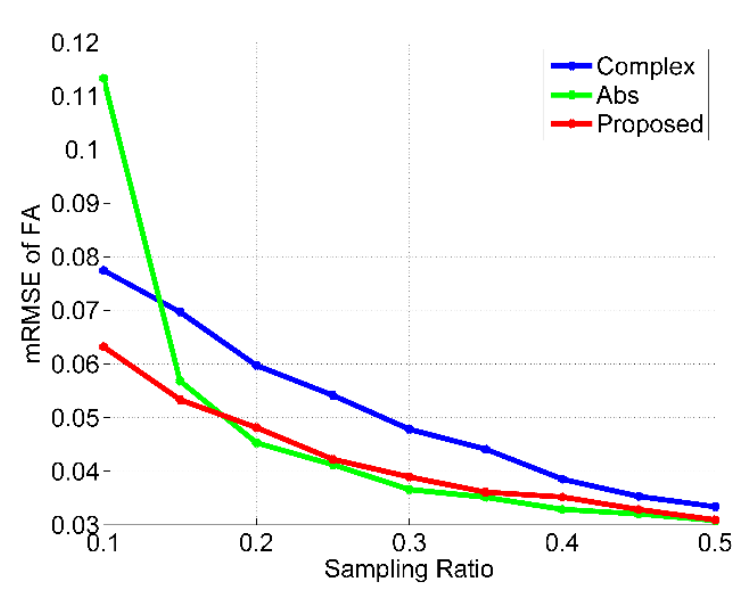

$\mathbf{a}$

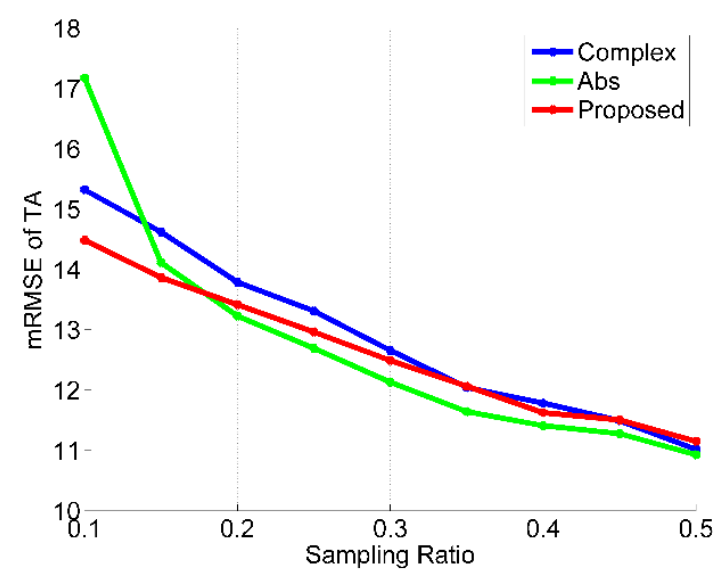

C

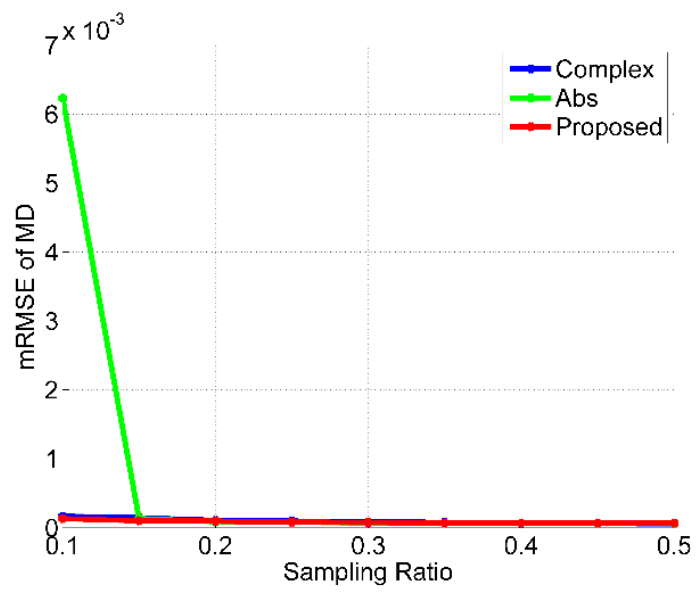

b

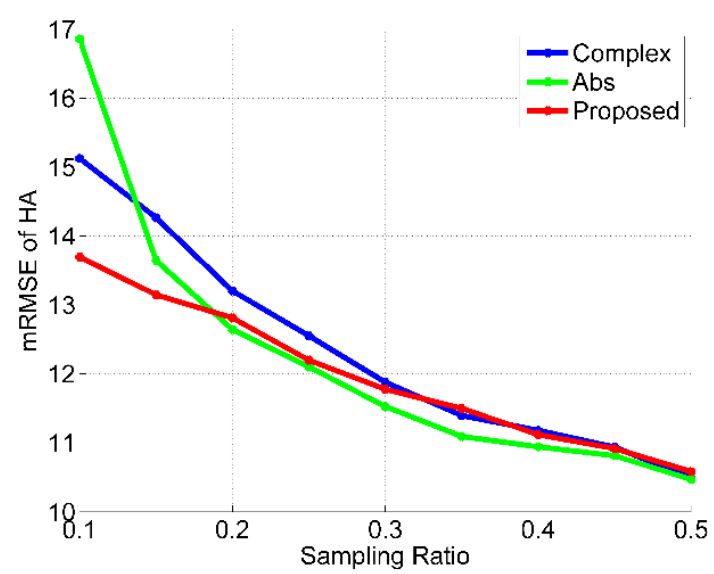

d

Fig. 144 Comparison of reconstructions using or without using phase information for ISNR=20 dB. (a) mRMSE of FA, (b) mRMSE of MD, (c) mRMSE of TA, and (d) mRMSE of HA. "Abs" no phase information is used. "Complex": phase information is used.

On the other hand, for a given acquisition, k-space data can be altered and highly asymmetric, or equivalently the formation of the phase can be very complicated. A number of factors, such as k-space sampling (random, irregular, etc.), noise, cardiac pulsations, respiratory motion, and multi-shot induced phase incoherence, can contribute to the formation of phase. Although the present study was also dealt with phase information, as shown in the above, but this phase stems from k-space undersampling and noise. In the future, it would be interesting to investigate multi-shot DWI reconstruction using explicit phase (by estimating for example low-resolution phase map from the fully sampled k-space data of each shot) or implicit phase (by using a structured low-rank matrix completion scheme [1]) to recover artifact-free images. Also, it is possible to undersample multi shot acquisitions using compressed sensing, the estimation of phase from the data would however be more complex and effective correction of motion-induced ghosting artifacts would be more difficult. More specifically for phase problems in cardiac DTI, there are phase changes not only in a given 
diffusion gradient direction (low variation) but also across diffusion gradient directions (drastic variation). It would then be interesting to study undersampled reconstruction based on phase correction [2], phase-constrained [3] or phase regularized reconstruction [4].

\subsection{Application to in vivo cardiac DTI}

The successful use of the proposed sampling-reconstruction method on in vivo cardiac DTI data would be conditioned by other two factors: motion correction and registration. Motion artifact may be due to cardiac and/or respiratory motion or any other phenomena such as arrhythmia. It is reflected by the presence of MRI signal loss. In in vivo DTI acquisitions, due to physical and technical limitations of MRI machines, all the images cannot be acquired at the same time point. Therefore, image registration is mandatory to correct for geometrical inconsistency among different scans. However, for a given in vivo DTI dataset, we are not able to determine the origin of errors in the calculated DTI indices if we do not separately consider sampling-reconstruction, motion and registration. In any case, if the sampling-reconstruction does not work, that will not make sense to investigate motion correction or image registration. However, motion correction and image registration in cardiac DTI still remain very challenging problems. Designing new imaging sequences less sensitive to motion artifacts, such as MUSE [67] and 3D - DISPENSE [68] techniques, could be an interesting way to explore. Once the problems are resolved, in vivo data would be rather close to the situation of ex vivo data.

Another particularity of in vivo cardiac DTI is the mixture of noise and artifacts due for example to motions. Noise designates electronics noise and thermal noise from subject while artifacts represent degradations due to motions. Therefore, once appropriate motion correction (and image registration to some extent) is achieved, in vivo data would be rather close to the situation of ex vivo data.

\section{Conclusion}

This work has proposed an efficient method for reconstructing DW images in DTI from highly undersampled k-space data using the LLR model and 3D TV constraints. The LLR model exploits the fact that the block matrices constructed from the set of DW images corresponding to different diffusion gradient directions are more strongly rank-deficient than the global matrix formed of entire DW images. The reconstruction using the combination of local low-rank penalty and 3D TV penalties thus enables us to improve reconstruction performance. The results on real human ex vivo 
cardiac DW images showed that, for a large range of sampling ratios from $10 \%$ to $50 \%$, the proposed method provides better reconstruction quality and more accurate DTI indices such as mean diffusivity, fractional anisotropy, helix angle and transverse angle, in comparison with the state-of-the-art CS-based DW image reconstruction methods. In the future work, we will apply the proposed method to in vivo cardiac DTI. To this end, phase correction, motion correction and image registration would have to be performed to obtain correct DTI indices.

\section{Acknowledgements}

This work was supported in part by the National Natural Science Foundation of China (no. 61701105, 61661010, 61601057), the Natural Science Foundation of Heilongjiang Province of China (no. QC2017066), the Nature Science Foundation of Guizhou province (Qiankehe J No.20152044), the Project funded by China Postdoctoral Science Foundation (no. 2017M610199), and the Program PHC-Cai Yuanpei 2018 ( ${ }^{\circ}$ 41400TC).

Author contribution statement The work presented in this paper corresponds to a collaborative development by all authors. Jianping Huang and Wanyu Liu conceived and designed the experiments; Jianping Huang and Lihui Wang performed the numerical experiments; Jianping Huang, Lihui Wang, Chunyu Chu and Yuemin Zhu analyzed the experimental results; Jianping Huang and Yuemin Zhu wrote the manuscript and improved this manuscript's English language and style.

Informed consent This manuscript does not contain clinical studies or patient data. The DW data of the human hearts in this study from the website http://cvrgrid.org/data/ex-vivo.

Conflict of interest The authors declare that they have no competing interests.

\section{References}

1. Froeling M, Strijkers G, Nederveen A, Chamuleau S, Luijten P (2014) Diffusion Tensor MRI of the Heart - In Vivo Imaging of Myocardial Fiber Architecture. Curr Cardiovasc Imaging Rep 7 (7):1-11. doi:10.1007/s12410-014-9276-y

2. Naumova AV, Yarnykh VL (2014) Assessment of Heart Microstructure From Mouse to Man. Circulation 129 (17):1720-1722. doi:10.1161/circulationaha.114.009221

3. Tournier J-D, Mori S, Leemans A (2011) Diffusion Tensor Imaging and Beyond. Magnetic Resonance in Medicine 65 (6):1532-1556. doi:10.1002/mrm.22924

4. Yang F, Zhu Y-M, Magnin IE, Luo J-H, Croisille P, Kingsley PB (2012) Feature-based 
interpolation of diffusion tensor fields and application to human cardiac DT-MRI. Medical Image Analysis 16 (2):459-481. doi:10.1016/j.media.2011.11.003

5. Basser PJ, Mattiello J, Lebihan D (1994a) MR diffusion tensor spectroscopy and imaging. Biophysical Journal 66 (1):259-267

6. Basser PJ, Mattiello J, Lebihan D (1994b) Estimation of the Effective Self-Diffusion Tensor from the NMR Spin-Echo. Journal of Magnetic Resonance Series B 103 (3):247-254. doi:10.1006/jmrb.1994.1037

7. Scollan DF, Holmes A, Winslow R, Forder J (1998) Histological validation of myocardial microstructure obtained from diffusion tensor magnetic resonance imaging. American Journal of Physiology-Heart and Circulatory Physiology 275 (6):H2308-H2318

8. Holmes AA, Scollan DF, Winslow RL (2000) Direct histological validation of diffusion tensor MRI in formaldehyde-fixed myocardium. Magnetic Resonance in Medicine 44 (1):157-161. doi:10.1002/1522-2594(200007)44:1<157::aid-mrm22>3.0.co;2-f

9. Mekkaoui C, Reese TG, Jackowski MP, Bhat H, Sosnovik DE (2015) Diffusion MRI in the heart. Nmr in Biomedicine:n/a-n/a. doi:10.1002/nbm.3426

10. Pravdin SF, Berdyshev VI, Panfilov AV, Katsnelson LB, Solovyova O, Markhasin VS (2013) Mathematical model of the anatomy and fibre orientation field of the left ventricle of the heart. Biomedical Engineering Online 12. doi:10.1186/1475-925x-12-54

11. Lopez-Perez A, Sebastian R, Ferrero JM (2015) Three-dimensional cardiac computational modelling: methods, features and applications. Biomedical Engineering Online 14. doi:10.1186/s12938-015-0033-5

12. Helm PA, Tseng HJ, Younes L, McVeigh ER, Winslow RL (2005) Ex vivo 3D diffusion tensor imaging and quantification of cardiac laminar structure. Magnetic Resonance in Medicine 54 (4):850-859. doi:10.1002/mrm.20622

13. Geerts L, Bovendeerd P, Nicolay K, Arts T (2002) Characterization of the normal cardiac myofiber field in goat measured with MR-diffusion tensor imaging. American Journal of Physiology-Heart and Circulatory Physiology 283 (1):H139-H145

14. Wu EX, Wu Y, Nicholls JM, Wang J, Liao S, Zhu S, Lau C-P, Tse H-F (2007) MR diffusion tensor imaging study of postinfarct myocardium structural remodeling in a porcine model. Magnetic Resonance in Medicine 58 (4):687-695. doi:10.1002/mrm.21350

15. Strijkers GJ, Bouts A, Blankesteijn WM, Peeters THJM, Vilanova A, van Prooijen MC, Sanders HMHF, Heijman E, Nicolay K (2009) Diffusion tensor imaging of left ventricular remodeling in response to myocardial infarction in the mouse. Nmr in Biomedicine 22 (2):182-190. doi:10.1002/nbm.1299

16. Yang F, Zhu YM, Rapacchi S, Luo JH, Robini M, Croisille P (2011) Interpolation of vector fields from human cardiac DT-MRI. Physics in Medicine and Biology 56 (5):1415-1430. doi:10.1088/0031-9155/56/5/013 
17. Dou JG, Tseng WYI, Reese TG, Wedeen VJ (2003) Combined diffusion and strain MRI reveals structure and function of human myocardial laminar sheets in vivo. Magnetic Resonance in Medicine 50 (1):107-113. doi:10.1002/mrm.10482

18. Wu M-T, Tseng W-YI, Su M-YM, Liu C-P, Chiou K-R, Wedeen VJ, Reese TG, Yang C-F (2006) Diffusion tensor magnetic resonance imaging mapping the fiber architecture remodeling in human myocardium after infarction - Correlation with viability and wall motion. Circulation 114 (10):1036-1045. doi:10.1161/circulationhaha.105.545863

19. Nielles-Vallespin S, Mekkaoui C, Gatehouse P, Reese TG, Keegan J, Ferreira PF, Collins S, Speier P, Feiweier T, de Silva R, Jackowski MP, Pennell DJ, Sosnovik DE, Firmin D (2013) In vivo diffusion tensor MRI of the human heart: Reproducibility of breath-hold and navigator-based approaches. Magnetic Resonance in Medicine 70 (2):454-465. doi:10.1002/mrm.24488

20. Nguyen C, Fan Z, Sharif B, He Y, Dharmakumar R, Berman DS, Li D (2014) In Vivo Three-Dimensional High Resolution Cardiac Diffusion-Weighted MRI: A Motion Compensated Diffusion-Prepared Balanced Steady-State Free Precession Approach. Magnetic Resonance in Medicine 72 (5):1257-1267. doi:10.1002/mrm.25038

21. Welsh CL, DiBella EVR, Hsu EW (2015) Higher-Order Motion-Compensation for In Vivo Cardiac Diffusion Tensor Imaging in Rats. IEEE Transactions on Medical Imaging 34 (9):1843-1853. doi:10.1109/tmi.2015.2411571

22. Dou JG, Reese TG, Tseng WYI, Wedeen VJ (2002) Cardiac diffusion MRI without motion effects. Magnetic Resonance in Medicine 48 (1):105-114. doi:10.1002/mrm.10188

23. Wei H, Viallon M, Delattre BMA, Wang L, Pai VM, Wen H, Xue H, Guetter C, Croisille P, Zhu Y (2013) Assessment of Cardiac Motion Effects on the Fiber Architecture of the Human Heart In Vivo. IEEE Transactions on Medical Imaging 32 (10):1928-1938. doi:10.1109/tmi.2013.2269195

24. Wei H, Viallon M, Delattre BMA, Moulin K, Yang F, Croisille P, Zhu Y (2015) Free-Breathing Diffusion Tensor Imaging and Tractography of the Human Heart in Healthy Volunteers Using Wavelet-Based Image Fusion. IEEE Transactions on Medical Imaging 34 (1):306-316. doi:10.1109/tmi.2014.2356792

25. Holdsworth SJ, Skare S, Newbould RD, Bammer R (2009) Robust GRAPPA-Accelerated Diffusion-Weighted Readout-Segmented (RS)-EPI. Magnetic Resonance in Medicine 62 (6):1629-1640. doi:10.1002/mrm.22122

26. Bammer R, Auer M, Keeling SL, Augustin M, Stables LA, Prokesch RW, Stollberger R, Moseley ME, Fazekas F (2002) Diffusion tensor imaging using single-shot SENSE-EPI. Magnetic Resonance in Medicine 48 (1):128-136. doi:10.1002/mrm.10184

27. Bammer R, Keeling SL, Augustin M, Pruessmann KP, Wolf R, Stollberger R, Hartung HP, Fazekas F (2001) Improved diffusion-weighted single-shot echo-planar imaging (EPI) in stroke 
using Sensitivity Encoding (SENSE). Magnetic Resonance in Medicine 46 (3):548-554. doi:10.1002/mrm.1226

28. Jaermann T, Crelier G, Pruessmann KP, Golay X, Netsch T, van Muiswinkel AMC, Mori S, van Zijl PCM, Valavanis A, Kollias S, Boesiger P (2004) SENSE-DTI at 3 T. Magnetic Resonance in Medicine 51 (2):230-236. doi:10.1002/mrm.10707

29. Larkman DJ, Nunes RG (2007) Parallel magnetic resonance imaging. Physics in Medicine and Biology 52 (7):R15-R55. doi:10.1088/0031-9155/52/7/r01

30. Hsu EW, Henriquez CS (2001) Myocardial fiber orientation mapping using reduced encoding diffusion tensor imaging. Journal of Cardiovascular Magnetic Resonance 3 (4):339-347. doi:10.1081/jcmr-100108588

31. Jiang Y, Hsu EW (2005) Accelerating MR diffusion tensor imaging via filtered reduced-encoding projection-reconstruction. Magnetic Resonance in Medicine 53 (1):93-102. doi:10.1002/mrm.20311

32. Lau AZ, Tunnicliffe EM, Frost R, Koopmans PJ, Tyler DJ, Robson MD (2015) Accelerated Human Cardiac Diffusion Tensor Imaging Using Simultaneous Multislice Imaging. Magnetic Resonance in Medicine 73 (3):995-1004. doi:10.1002/mrm.25200

33. Taron J, Martirosian P, Schwenzer NF, Erb M, Kuestner T, Weiss J, Othman A, Notohamiprodjo M, Nikolaou K, Schraml C (2016) Scan time minimization in hepatic diffusion-weighted imaging: evaluation of the simultaneous multislice acceleration technique with different acceleration factors and gradient preparation schemes. Magnetic Resonance Materials in Physics Biology and Medicine 29 (5):739-749. doi:10.1007/s10334-016-0553-4

34. Candes EJ, Romberg J, Tao T (2006) Robust uncertainty principles: Exact signal reconstruction from highly incomplete frequency information. IEEE Transactions on Information Theory 52 (2):489-509. doi:10.1109/tit.2005.862083

35. Donoho DL (2006) Compressed sensing. IEEE Transactions on Information Theory 52 (4):1289-1306. doi:10.1109/tit.2006.871582

36. Candes EJ, Tao T (2006) Near optimal signal recovery from random projections: Universal encoding strategies? IEEE Transactions on Information Theory 52 (12):5406-5425. doi:10.1109/tit.2006.885507

37. Graff CG, Sidky EY (2015) Compressive sensing in medical imaging. Applied Optics 54 (8):C23-C44. doi:10.1364/ao.54.000c23

38. Wang G, Bresler Y, Ntziachristos V (2011) Compressive Sensing for Biomedical Imaging. IEEE Transactions on Medical Imaging 30 (5):1013-1016. doi:10.1109/tmi.2011.2145070

39. Lustig M, Donoho DL, Santos JM, Pauly JM (2008) Compressed sensing MRI. IEEE Signal Processing Magazine 25 (2):72-82. doi:10.1109/msp.2007.914728

40. Hollingsworth KG (2015) Reducing acquisition time in clinical MRI by data undersampling and compressed sensing reconstruction. Physics in Medicine and Biology 60 (21):R297-R322. 
doi:10.1088/0031-9155/60/21/r297

41. Chen S, Du H, Wu L, Jin J, Qiu B (2017) Compressed sensing MRI via fast linearized preconditioned alternating direction method of multipliers. Biomedical Engineering Online 16. doi:10.1186/s12938-017-0343-x

42. Ritschl L, Sawall S, Knaup M, Hess A, Kachelriess M (2012) Iterative 4D cardiac micro-CT image reconstruction using an adaptive spatio-temporal sparsity prior. Physics in Medicine and Biology 57 (6):1517-1525. doi:10.1088/0031-9155/57/6/1517

43. Hu Z, Zheng H (2014) Improved total variation minimization method for few-view computed tomography image reconstruction. Biomedical Engineering Online 13. doi:10.1186/1475-925x-13-70

44. Rigie DS, La Riviere PJ (2015) Joint reconstruction of multi-channel, spectral CT data via constrained total nuclear variation minimization. Physics in Medicine and Biology 60 (5):1741-1762. doi:10.1088/0031-9155/60/5/1741

45. Adluru G, Hsu E, Di Bella EVR (2007) Constrained reconstruction of sparse cardiac MR DTI data. Paper presented at the Functional Imaging and Modeling of the Heart, Proceedings, Salt Lake, USA, June

46. Wu Y, Zhu Y-J, Tang Q-Y, Zou C, Liu W, Dai R-B, Liu X, Wu EX, Ying L, Liang D (2014) Accelerated MR Diffusion Tensor Imaging Using Distributed Compressed Sensing. Magnetic Resonance in Medicine 71 (2):763-772. doi:10.1002/mrm.24721.

47. Shi X, Ma X, Wu W, Huang F, Yuan C, Guo H (2014) Parallel imaging and compressed sensing combined framework for accelerating high-resolution diffusion tensor imaging using inter-image correlation. Magnetic Resonance in Medicine

48. Hao Gao, Li L, Hu aX (2013) Compressive Diffusion MRI- Part 1 Why Low-Rank. Paper presented at the Proceedings of the 21th Annual Meeting of ISMRM, Salt Lake, April

49. Ma S, Nguyen C, Christodoulou A, Luthringer D, Kobashigawa J, Lee S-E, Chang H-J, Li D (2017) Accelerated Cardiac Diffusion Tensor Imaging Using Joint Low-Rank and Sparsity Constraints. IEEE transactions on biomedical engineering. doi:10.1109/tbme.2017.2787111

50. Gao H, Li L, Zhang K, Zhou W, Hu X (2014) PCLR: Phase-Constrained Low-Rank Model for Compressive Diffusion-Weighted MRI. Magnetic Resonance in Medicine 72 (5):1330-1341. doi:10.1002/mrm.25052

51. Welsh CL, DiBella EVR, Adluru G, Hsu EW (2013) Model-based reconstruction of undersampled diffusion tensor k-space data. Magnetic Resonance in Medicine 70 (2):429-440. doi:10.1002/mrm.24486

52. Zhu Y, Wu Y, Zheng Y, Wu EX, Ying L, Liang D (2012) A model-based method with joint sparsity constraint for direct diffusion tensor estimation. 2012 IEEE 9th International Symposium on Biomedical Imaging:510-513. doi:10.1109/isbi.2012.6235597

53. Dong Z, Dai E, Wang F, Zhang Z, Ma X, Yuan C, Guo H (2018) Model-based reconstruction for 
simultaneous multislice and parallel imaging accelerated multishot diffusion tensor imaging. Medical Physics 45 (7):3196-3204. doi:10.1002/mp.12974

54. Lugauer F, Nickel D, Wetzl J, Kiefer B, Hornegger J, Maier A (2017) Accelerating multi-echo water-fat MRI with a joint locally low-rank and spatial sparsity-promoting reconstruction. Magnetic Resonance Materials in Physics Biology and Medicine 30 (2):189-202. doi:10.1007/s 10334-016-0595-7

55. Trzasko J, Manduca A, Borisch E (2011) Local versus global low-rank promotion in dynamic MRI series reconstruction. Paper presented at the Proc. Int. Symp. Magn. Reson. Med,

56. Zhang T, Pauly JM, Levesque IR (2015) Accelerating Parameter Mapping with a Locally Low Rank Constraint. Magnetic Resonance in Medicine 73 (2):655-661. doi:10.1002/mrm.25161

57. Huang J, Zhang S, Metaxas D (2011) Efficient MR image reconstruction for compressed MR imaging. Medical Image Analysis 15 (5):670-679. doi:10.1016/j.media.2011.06.001

58. Beck A, Teboulle M (2009) A Fast Iterative Shrinkage-Thresholding Algorithm for Linear Inverse Problems. SIAM Journal on Imaging Sciences 2 (1):183-202. doi:10.1137/080716542

59. Helm PA, Younes L, Beg MF, Ennis DB, Leclercq C, Faris OP, McVeigh E, Kass D, Miller MI, Winslow RL (2006) Evidence of structural remodeling in the dyssynchronous failing heart. Circulation research 98 (1):125-132. doi:10.1161/01.RES.0000199396.30688.eb

60. Helm P, Beg MF, Miller MI, Winslow RL (2005) Measuring and Mapping Cardiac Fiber and Laminar Architecture Using Diffusion Tensor MR Imaging. Annals of the New York Academy of Sciences 1047 (1):296-307. doi:10.1196/annals.1341.026

61. Lustig M, Donoho D, Pauly JM (2007) Sparse MRI: The application of compressed sensing for rapid MR imaging. Magnetic Resonance in Medicine 58 (6):1182-1195. doi:10.1002/mrm.21391

62. Tsai CM, Nishimura DG (2000) Reduced aliasing artifacts using variable-density k-space sampling trajectories. Magnetic Resonance in Medicine 43 (3):452-458. doi:10.1002/(sici)1522-2594(200003)43:3<452::aid-mrm18>3.0.co;2-b

63. Streeter DD, Jr., Spotnitz HM, Patel DP, Ross J, Jr., Sonnenblick EH (1969) Fiber orientation in the canine left ventricle during diastole and systole. Circulation research 24 (3):339-347

64. Carrillo RE, McEwen JD, Wiaux Y (2012) Sparsity Averaging Reweighted Analysis (SARA): a novel algorithm for radio-interferometric imaging. Monthly Notices of the Royal Astronomical Society 426 (2):1223-1234. doi:10.1111/j.1365-2966.2012.21605.x

65. Lagae A, Dutre P (2008) A comparison of methods for generating Poisson disk distributions. Computer Graphics Forum 27 (1):114-129. doi:10.1111/j.1467-8659.2007.01100.x

66. Marseille GJ, deBeer R, Fuderer M, Mehlkopf AF, vanOrmondt D (1996) Nonuniform phase-encode distributions for MRI scan time reduction. Journal of Magnetic Resonance Series B 111 (1):70-75. doi:10.1006/jmrb.1996.0061

67. Chen N-k, Guidon A, Chang H-C, Song AW (2013) A robust multi-shot scan strategy for high-resolution diffusion weighted MRI enabled by multiplexed sensitivity-encoding (MUSE). 
Neuroimage 72:41-47. doi:10.1016/j.neuroimage.2013.01.038

68. Zhang Q, Coolen BF, Nederveen AJ, Strijkers GJ (2019) Three-dimensional diffusion imaging with spiral encoded navigators from stimulated echoes (3D-DISPENSE). Magnetic Resonance in Medicine 81 (2):1052-1065. doi:10.1002/mrm.27470 